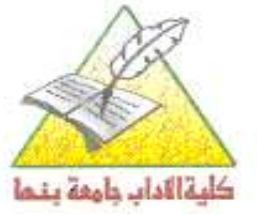

مجلة كلية الآداب

بلة دورية علمية محكة

نصف سـنوية

المدد الثامن والالربمون

اكتوبر r.v 


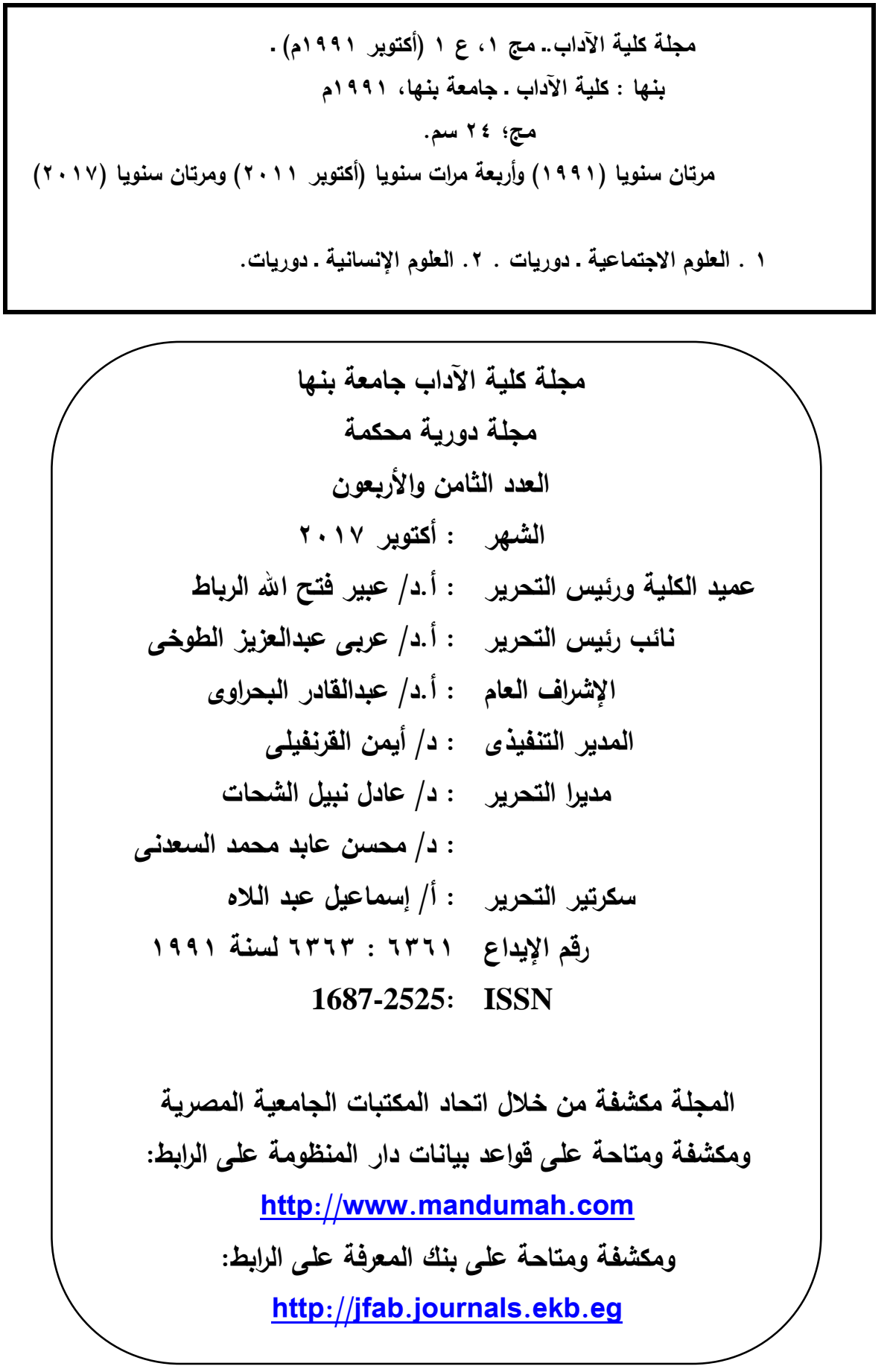




\section{هينّة تحرير الطجلة}

$$
\begin{aligned}
& \text { عميد الكلية ورئيس مجلس الإدارة } \\
& \text { ورئيس التحرير } \\
& \text { أ.د/عبرفتحالهالراط } \\
& \text { نائب رئيس التحرير }
\end{aligned}
$$

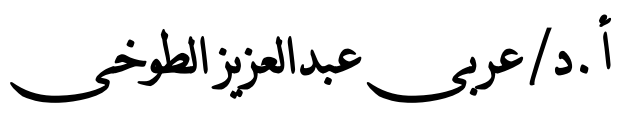

$$
\begin{aligned}
& \text { الإشراف العام } \\
& \text { أ .د /عبدالقادر البحراوى } \\
& \text { المدير التنفيذى }
\end{aligned}
$$

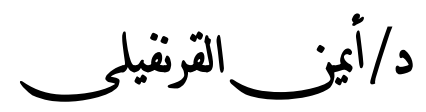

$$
\begin{aligned}
& \text { مدير تحرير المجلة } \\
& \text { د/عادلنبيل } \\
& \text { مدير تحرير المجلة }
\end{aligned}
$$

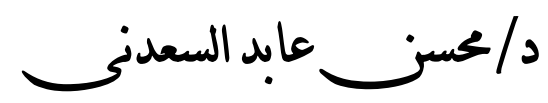

$$
\begin{aligned}
& \text { سكرتير التحرير } \\
& \text { آ/إسماعيل عبد اللاه }
\end{aligned}
$$




\title{
Marie Curie \\ Un Modèle de maîtrise de soi
}

\author{
Recherche présentée \\ Par \\ Dr/ Abir chéhata
}


"( (....) La vie, à ce qu'il semble, n'est facile pour aucun de nous. Mais quoi, il faut avoir de la persévérance et surtout de la confiance en soi !, Il faut croire que l'on est doué pour quelque chose et que cette chose, il faut donc

l'atteindre coûte que coûte.»

(Lettre de Marie Curie à son frère Joseph le 18 mars 1894) 


\section{Introduction}

Regroupant des documents, la biographie demeure souvent le fait des chercheurs ainsi que d'érudits dont la démarche s'apparente à celle des historiens. Furetant dans le passé des hommes et des femmes dont ils décrivent la vie, ceux-ci s'intéressent aux faits qui ont marqué cette dernière.Ils confient alors des récits détaillés visant à une loyale restitution de la vérité.

$$
\begin{aligned}
& \text { "Parler du «biographique », c'est } \\
& \text { évoquer tout ce qui est énonciation } \\
& \text { textuelle ou orale, littéraire ou non } \\
& \text { littéraire, d'une vie » }{ }^{(1)}
\end{aligned}
$$

Dés L'origine, la biographie projette le récit d'une vie illustre: celle d'un grand personnage qui a effectivement existé et dont les actions méritent de rester imprimées dans la mémoire collective. Il s'agit donc d'une vie exemplaire au sens large du terme c'est à dire d'un modèle à suivre.

Femme de lettres, journaliste, conférencière et diplomate française, Eve Curie est la fille cadette de Pierre Curie et Marie Sklodowska. Elle étudie au Collège Sévigné où elle obtient son baccalauréat.Autonome et volontaire, Eve n'a pas définitivement songé à devenir scientifique.

Musicienne habile, elle commence sa carrière en tant que pianiste et se montre pour la première fois à Paris en 1925 puis elle se voue aux études littéraires et à L'écriture. Elle écrit alors «Madame Curie » livre sur la vie de sa mère, publié en 1937 trois ans après la mort de celle-ci. Cette biographie est considérée comme un classique parmi les biographies des scientifiques. En outre, elle est devenue un succès mondial de librairie en 1938. Ce succès ne vient jamais du vide, il vient certes de la façon de l'écrivain Eve Curie de livrer à ses lecteurs la vérité sans rien leur

1) Maillard, (M), L'autobiographie et la biographie Nathan/VUEF-2001, P.4 Vol. 48 
cacher même sans rien leur taire comme elle le déclare ellemême

"(...) Je n'ai pas relaté une seule
anecdote dont je ne sois sûre. Je n'ai
pas déformé une phrase essentielle,
inventé la couleur d'une robe, les
faits ont eu lieu, les paroles ont été
prononcées. ${ }^{(1)}$

La double volonté de transparence et de sincérité est indissociable d'une recherche de la conformité. Or ce récit biographique est mis en accord avec la réalité vécue. Le respect de cet élément de conformité a donné toute son authenticité au récit de vie.

Les lecteurs joueront donc un rôle primordial dans l'expression de cette vérité puisqu'ils en seront les témoins nécessaires auquels se confie l'écrivain.

Cette biographie exhaustive est composée d'une introduction, de trois parties et enfin d' une appendice. La première partie se divise en sept chapitres racontant l'enfance, l'adolescence de Marie jusqu'à son départ à Paris.La deuxième partie se divise en onze chapitres montrant ses études à la Sorbonne, sa rencontre avec Pierre Curie, leur mariage, la découverte du Radium, sa thèse de doctorat ainsi que la mort de son compagnon.La troisième partie se divise en neuf chapitres relatant sa participation à la Première Guerre Mondiale, ses succès, ses épreuves et son voyage en Amérique jusqu'à la fin de sa mission. Quant à l'appendice, elle expose une liste énorme des prix décernés à madame Curie.

Dés le début de l'introduction, nous lisons cette phrase qui attire notre attention.

1) Curie, (E) Madame Curie, Libreria Hachette S.A. Buenos Aires, P.8 Vol. 48 
«Il ya, dans la vie de Marie Curie, un tel nombre de grands traits, que l'on voudrait conter son histoire comme une légende. »

Puis nous suivons passionnément les petites et les grandes lignes de la vie d'un être remarquable, d'un rare génie qui a réussi à réaliser son rêve malgré les nombreuses difficultés vécues. Il s'agit en effet d'une riche vie laborieuse où nous apprenons une véritable leçon exemplaire de Maîtrise de Soi.

\section{Naissance de Marya Sklodowska}

La Petite «Mania » naît le 7 novembre 1867 à Varsovie en Pologne . Son père, Wladyslaw Sklodowski est professeur de physique et sous-inspecteur au gymnase de la ville. Quant à sa mère, elle occupe un poste de directrice d'école mais elle démissionne pour s'occuper de ses cinq enfants.

Lors de la naissance de Mania, la Pologne connaît depuis de nombreuses années une tyrannie étrangère. Des rois avides, terribles voisins de ce pays épuisée ont décidé sa perte en divisant sa terre déchirante.Les Polonais se sont révoltés contre leurs ennemis mais ceux- $\mathrm{Ci}$ ne possèdent que « des piques, des faux et des gourdins à opposer aux carabines tsaristes $»^{(2)}$

Des convois de rebelles enchaînés sont condamnés aux travaux forcés en Sibérie arrivant même à les pendre au sommet du rempart la ville. Le tsar Nicolas a imposé en Pologne « russe »de strictes mesures de représailles.

« Surveiller les Polonais, persécuter leur religion, interdire les journaux et les livres suspects, abolir peu à peu l'usage de la langue (3)

1) Curie, (E) Madame Curie, opcit., P.8

2) Ibid., P.22

3) Curie, (E) Madame Curie, opcit., P.22

Vol. 48 
Ainsi toutes les mesures ont été prises pour réduire à l'obéissance la Pologne qui n'accepte jamais de mourir. Sans doute, cette atmosphère a influencé les enfants du professeur Sklodowski qui, de cinq à neuf ans, jouent à la guerre en poussant des cris effrayants avec des cubes de bois offerts par leur oncle un soir de Noël.

"(...) de courtes colonnes de chêne forment l'artillerie, les petits cubes servent de boulets, et les architectes sont devenus des maréchaux. » (1)

La petite Mania aime bien jouer avec ses sœurs et son frère mais ce qu'elle préfère c'est asseoir à côté de sa sœur aînée "Zosia » qui lui raconte pendant des heures à haute voix de belles histoires ou bien elle regarde des tubes de verre, des balances, de petits morceaux de minéraux et même un électroscope, objets emportés par son père de la salle de classe pendant les cours.

"Mania ne peut imaginer à quoi servent ces passionnants bibelots. Un jour que, dressée sur la pointe des pieds, elle les contemplait avec béatitude, son père lui a simplement dit leur nom : "AP-PA-REILS-DE-PHY-SI QUE» Un drôle de nom.

Elle ne l'a pas oublié - elle n'oublie jamais rien $\|^{(2)}$

Très tôt, la petite Mania montre un goût pour l'étude. Ses parents, en tant que pédagogues sages, appréhendent la précocité de leur enfant prodige et évitent toujours de lui laisser des livres et à chaque fois qu'elle tend sa main pour en prendre un traînant dans la maison, une voix lui conseille de chanter ou d'aller dans le jardin.

" (...) dans chaque lycée de Pologne, un antagonisme profond oppose, sous une

1) Ibid., P.14

2) Ibid., P.25

Vol. 48 
courtoisie affecteé, les vaincus aux vainqueurs, les professeurs brimés aux directeurs espions-les sklodowski aux Iwanow.» ${ }^{(1)}$

\section{Une jeunesse difficile dans un pays asservi}

Écolière de 10 ans, Marya Sklodowska se distingue de ses camarades en récitant ses leçons d'une jolie voix assurée .L'uniforme "en serge bleu marine, boutonné d'acier, au col blanc bien empesé, engonce la silhouette de cette fillette ${ }^{(2)}$ paraît si rigide ainsi que la coiffure sévère telle est la loi de l'école privée de Mlle Sikorska.

Quant à Mlle Antonine Tupalska, la maîtresse d'arithmétique et d'histoire, à la figure laide et brutale, elle exerce aussi les tâches de surveillante. En cet attribut, elle a pu sévir contre l'esprit autonome et le caractère tenace de la fillette Marya, mais il y a cependant dans son regard beaucoup d' affection c'est pourquoi on la choisit souvent pour l'interrogatoire de M. Hornberg, l'inspecteur des pensionnats privés.

Après avoir récité « Notre père » et dit tous les noms des Tsars qui ont régné sur la Russie, celui-ci est satisfait de sa bonne mémoire et de sa merveilleuse prononcitation du russe.

"Une des humiliations les plus subtiles qu'ait découvertes le Tsar est d'obliger les enfants polonais à dire chaque jour en russe les prières de la religion catholique. Ainsi, prétendant respecter leurs croyances, il leur fait profaner ce qu'ils vénèrent. " ${ }^{(3)}$

Plusieurs heures se sont écoulées de son interrogatoire mais la fillette en est encore agitée. Elle déteste ces présentations paniques subites où il faut mentir.À cause de cette visite, elle sent

1) Curie, (E) Madame Curie, opcit., P.23

2) Ibid., P.26

3) Curie, (E) Madame Curie, opcit., P.29

Vol. 48 
de plus en plus lourde les difficultés de sa vie or de pires catastrophes successives se sont abattues sur sa famille. Mme sklodowska a été frappée d'une grave maladie. Les signes de la tuberculose sont apparus chez elle et en dépit des cures, ce mal a fait une sûre progression. De suite, elle s'est imposée des règles sévères : se servir d'une vaisselle à elle seule, ne pas embrasser ni son fils ni ses filles. En automne 1873, M. Sklodowski a trouvé sur son bureau une lettre officielle : par ordre des autorités, ses gages étaient réduits, son logement de fonctionnaire lui était repris ainsi que son titre de sous-inspecteur.

Après avoir déménagé, les Sklodowski se sont installés dans un appartement au coin de la rue Nowolipki et de la rue des Carmélites. Et leur vie a subi peu à peu des modifications qui reflètent le dérangement, l'embarras financier. Le professeur a d'abord pris deux ou trois pensionnaires puis cinq, huit, dix choisis parmi ses élèves, il leur donne le logement, la nourriture et des leçons particulières. Ainsi l'intimité de la maison est perdue. En plus, excité par un déporable beau-frère dans une transaction hasardeuse, ce professeur, à l'accoutumée prudent, a perdu trente mille roubles qui représentaient toutes ses économies ce qui l'emmène à dire.

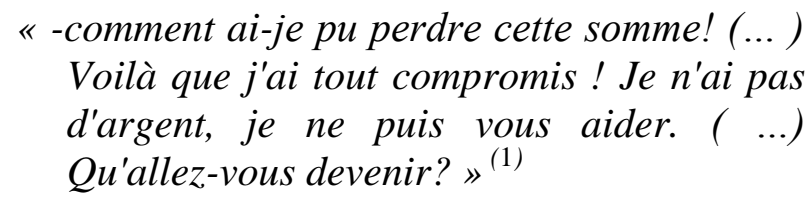

Un malheur insupportable est arrivé à Mania: celui de la maladie de ses deux sœurs Bronia et Zosia. Celles-ci sont infectées par le typhus, une maladie contagieuse.Dans une chambre, la mère tentait de retenir ses toux. Dans une autre, les deux sœurs souffraient de fièvre.En Janvier 1876, C'était la première rencontre de Mania avec la mort en voyant sa sœur aînée «Zosia»

1) Ibid., PP.59-60

Vol. 48 
étalée dans le cercueil et c'était de même le premier convoi qu'elle a accompagné, vêtue d'un morne petit manteau noir.Deux ans plus tard, exactement en maï 1878 , la mère meurt après avoir dit adieu à son mari et ses enfants.De nouveau, vêtue de noir, elle ne peut imaginer "ce qu'une intendante, engagée à la hâte par le professeur, vienne chaque jour donner des ordres aux servantes, composer le menu des pensionnaires et surveiller distraitement la toilette des enfants » (1).

Privée, de très bonne heure ,de la tendresse maternelle ainsi que de la protection de la sœur aînée, Mania apprend tôt l'idée de la dureté et la cruauté de la vie pour les êtres humains. En dépit du tribut payé au malheur, les quatre enfants Bronia, Hela, Joseph et Mania portent en eux une énergie invincible. La jeunesse de Marya Sklodowska est obsédée par trois mots : Gymnases, pensionnats et écoles. Selon elle, l'univers se présente comme un énorme lycée où il n'existe que des professeurs, des élèves et où domine une seule idée : Apprendre.

À quatorze ans, Mania est l'élève douée dans le même gymnase où Bronia a terminé ses études en remportant une médaille d'or. Une deuxième médaille d'or pareille à celle de Bronia a été accordée à Joseph lorsque celui-ci a quitté le gymnase de garçons pour suivre ses cours à la faculté de Médecine. La troisième est attribuée à Mania le 12 juin 1883, date de la fin de ses études secondaires.

Tenaillées par des rêves épisodiques, les deux sœurs haïssent le règlement de l'université de Varsovie où la rentrée des femmes n'est pas admise.

\section{Signes de la patience}

$$
\begin{gathered}
\text { "(...) la patience est cette vertu qui } \\
\text { permet d'attendre paisiblement }
\end{gathered}
$$

1) Curie, (E) Madame Curie, opcit., P.37

Vol. 48 
l'accomplissement des choses que nous espérons » ${ }^{(1)}$

M.Sklodowski a décidé qu'avant de choisir une carrière, Mania va vivre à la campagne chez des parents de province. Ils l'ont logé pendant un an en échange des leçons qu'elle donne à leurs enfants ou du paiement d'une pension modique.

Dans une lettre à son amie unique Kazia , elle raconte ainsi, ces séjours.

«(...) Je n'ai aucun emploi du temps fixe ( ....) Je ne lis aucun ouvrage sérieux, rien que des petits romans anodins et absurdes. Aussi, malgré le diplôme qui me confère la dignité et la maturité d'une personne qui a terminé ses études, je me sens incroyablement bête. "(2)

Grâce à son père, Mania a évolué dans une ambiance intellectuelle que connaissent peu de jeunes filles. Un fort lien l'attache à ce père qui fait de si émouvants efforts pour que sa vie soit intéressante et séduisante. Avec une maigre retraite, le chef de famille ne peut que payer le loyer, la nourriture, la servante, alors, les jeunes Sklodowski sont obligés de gagner leurs vies.

La patience est une vertu permettant en plus « de supporter les situations difficiles, la souffrance, l'épreuve, l'adversité, les personnes irritantes. Elle n'est pas la resignation, mais au contraire elle permet de persévérer, [d'] attendre et [de] travailler, car elle tend vers un résultat. Elle est étroitement associée à la foi dans laquelle elle permet de persévérer.» ${ }^{(3)}$

Essayant de sa part de résoudre le problème financier de son père, Mania a décidé de donner des leçons. Elle a connu ainsi l'épuisement et l'humiliation qui attendait une répétitrice : les longs pas à travers la ville, par le froid et la pluie, les élèves

1) www.pasteurweb.org/Etudes/le\%2520 Fruit \% 2520 de \% $25201 \% 27$ Esprit / la patience. Htm

2) Curie, (E) Madame Curie, opcit., P.49

3) www.pasteurweb.org/Etudes/le\%2520 Fruit \% 2520 de \% $25201 \% 27$ Esprit / la patience. Htm

Vol. 48 
rebelles et indolentes, les parents d'élèves qui vous font attendre sans fin ou qui oublient à la fin du mois de vous payer les quelques roubles qu'ils vous doivent.

Outre que cette pauvre vie indigente des leçons privées Mania a une autre vie discrète comme chaque polonaise patriotique de son temps, elle est enthousiasmée par des rêves.

" (...) on peut faire preuve de patience en réponse à un désir, dans l'espérance d'obtenir finalement ce dont l'accès immédiat est impossible ou dangereux. ( ...) le but à atteindre étant précisément celui d'un calme intérieur où l'attente, le désir, l'agitation, bref le futur et la projection personnelle sont oubliés. »(1)

Le rêve commun à tous les jeunes est le rêve national : le dessein de servir la Pologne passe dans leurs plans d'avenir avant l'ambition personnelle avant même l'amour et le mariage. Bien que Mania s'est comptée parmi ses amies des patriotes révolutionnaires auxquelles elle confiait son passeport, elle ne rêvait jamais de participer «à des attentats, de jeter des bombes sur la voiture du Tsar ou sur celle du Gouverneur de la ville. (...) Une seule chose compte : travailler, constituer à la Pologne un magnifique capital intellectuel et développer l'éducation du peuple, que les autorités maintiennent, exprès, dans l'obscurantisme» ${ }^{(2)}$

Le courage comme effort patient

Il est important de s'arrêter à l'écoute de Jankélévitch dans le traité des vertus

" l'intelligence nous conduit le plus loin possible dans la voie de la décision, mais il y a des choses qu' elle ne peut faire à la place du courage; elle ne saurait remplacer l'instant irremplaçable; elle ne

1) www.pasteurweb.org/Etudes/le\%2520 Fruit \% 2520 de \% $25201 \% 27$ Esprit / la patience. Htm

2) Curie, (E) Madame Curie, opcit., P.62

Vol. 48 
nous dispense pas de ce fiat vertigineux qui est la seule cause suffisante et décisive de l'acte ${ }^{(1)}$

Entourée par ses sœurs Bronia, Hela et une camarade, Marya a été admise aux cours de "L'université volante ». c'était de multiples leçons données par des professeurs bénévoles aux jeunes qui aspiraient à développer leur culture. Ces cours avaient lieu en cachette chez Mlle Piasecka ou dans quelque logis Privé.

" ( .....) Le courage est, comme la morale, $d u$ côté de la résistance, c'est une manière singulière de se poser et de s'opposer ( ....) à soi-même. Se déterminer, c'est d'abord combattre l'indétermination et la détermination machinale ou extérieure; la condition de cette détermination de soi réside dans une certaine "force» d'âme qui définit précisément le courage ${ }^{(2)}$

Groupés par huit ou dix les disciples prenaient des notes. Ils se communiquaient des opuscules et des articles. Le moindre bruit les frissonnaient. S'ils étaient trouvés par la police, c'était pour eux tous, la prison.

"le courage "est de supporter sans fléchir les épreuves de tout ordre, physiques et morales, que prodigue la vie. "(3)

Ajoutons encore que celui «qui est courageux, ce n'est donc plus l'action isolée ,mais la disposition générale face à la vie ${ }^{(4)}$

Encouragée par Mlle Piasecka , Marya a donné des leçons à des femmes du peuple. Elle a fait la lecture aux employées d'un

1) Jankélévitch, (V), Traité des vertus, « le courage et la fidélité », Paris, champs flammarion, 1985. P.380

${ }^{2)}$ Gaëlle, Jeanmart, les morales du courage- le courage des Anciens , http://www.philopol.ulg. Ac. Be/telechahger/.../ courage.P.2

3) Jaurès (J) ; Discours à la jeunesse - Albi - 1903

4) Gaëlle, Jeanmart, les morales du courage- le courage des Anciens, http://www.philopol.ulg. Ac. Be/telechahger/.../ courage.P.15

Vol. 48 
atelier de prêt- À- porter en constituant tome par tome, une petite bibliothèque, à la volonté des ouvrières.

«(...) Agir avec courage représente le
meilleur de l'intuition. C'est écouter
ce qui est centré avec noblesse, alors
que la peur anéantit toute intuition.»
(1)

De même, il est à noter que «l'attitude courageuse suit naturellement et sans faille la claire connaissance du devoir à accomplir .... " ${ }^{(2)}$

Non seulement se préoccuper de sa patrie russifiée asservie mais Marya ,la bienveillante s'est sentie responsable de l'avenir de ses aînés .Joseph et Hela lui ont donné peu de soucis :Joseph va devenir médecin ,la superbe Hela, hésitant entre le professorat et la carrière de chanteuse professionnelle obtient des diplômes en refusant les demandes en mariage .Mais comment aider Bronia?

Quand celle-ci a quitté le lycée, tous les soucis du ménage se sont précipités sur elle. A force d'acheter les aliments ,d'agencer les menus, Bronia est devenue une considérable maîtresse de maison et cette situation l'a chagrinée.

$$
\begin{aligned}
& \text { «(...) le courage est le mécanisme } \\
& \text { psychologique (...) qui procure la force, la } \\
& \text { clarté et le cran de triompher des obstacles. } \\
& \text { Il vous donne l'essor vers la lumière.» }{ }^{(3)}
\end{aligned}
$$

Mania a deviné l'amertume de sa sœur Bronia dont le grand rêve est d'aller à Paris faire ses études de médecine puis de retourner en Pologne travailler à la campagne.

1) Oroloff,Judith , http://www.eveil de la conscience. Ca/orloff $175208 \mathrm{htm}$. 18/10/2015

2) Gaëlle, Jeanmart, les morales du courage- le courage des Anciens, http://www.philopol.ulg. Ac. Be/telechahger/.../ courage.P.23

3) Oroloff, Judith, http://www.eveil de la conscience. Ca/orloff $175208 \mathrm{htm}$. 18/10/2015. page. 2

Vol. 48 
La pauvre Bronia a bien économisé mais un séjour à l'étranger coûte cher combien d'années faudra-t- il qu'elle patiente encore? L'irritation et le désespoir de sa sœur Bronia deviennent la préoccupation de Mania.

$$
\begin{aligned}
& \text { "(..) Celui qui ne rencontre nulle résistance } \\
& \text { n'aura pas connu " ce purgatoire de la } \\
& \text { résistance surmontée sans lequel la vertu } \\
& \text { ne peut-être méritoire }{ }^{(1)}
\end{aligned}
$$

De suite, le mérite réside de la somme des efforts acquiescés et donc au courage dont il a fallu faire preuve pour persister dans l'effort.

Dans une famille très homogène, les deux sœurs se sont choisies: la nature de l'une complète l'autre d'une manière étrange .L'ainée Bronia, par son expérience, en dicte à Mania qui lui soumet les moindres problèmes de la vie quotidienne.La cadette Mania ,plus ardente, est pour Bronia , une jeune compagne prodigieuse en qui l'amitié s'enrichit d'un sentiment de reconnaissance.

" (... ) le courage ne dépend pas seulement de la situation objective et de l'estimation des risques et des chances de succès, il est aussi et surtout dépendant de la valeur pour laquelle on est prêt à courir un risque.» ${ }^{(2)}$

pour résoudre le problème de Bronia ,Mania a tant réfléchi jusqu'à trouver un moyen essayant de faire comprendre à sa sœur.

$$
\begin{aligned}
& \text { "(...)Au début ,tu dépenseras ton } \\
& \text { argent.Ensuite,je m'arrangerai pour t'en } \\
& \text { envoyer; père aussi . En même temps } \\
& \text {,j'accumulerai des fonds pour mes études } \\
& \text { futures. Lorsque tu seras docteur,je partirai } \\
& \text { à mon tour.» }{ }^{(3)}
\end{aligned}
$$

1) Jankélévitch, Traité des vertus, Opcit., p.365

2) Gaëlle , (J), les morales du courge. Le courage des Anciens, http.www. philopol. ulg.ac.be/telecharger/.../courage p.19

3) Curie, (E) Madame Curie, opcit., P.67

Vol. 48 
Mais dans ce «fameux moyen», il y a un point qui est resté confus. C'était que Mania va se placer comme institutrice dans une famille vécue, sustentée, blanchie avec quatre cents roubles par an

Selon Robin S .Sharma «((...) le courage te permet de faire tout ce que tu veux parce que tu sais que c'est juste. Le courage te donne la maîtrise de toi - même

\section{Savoir maîtriser soi-même} pour persister ... ${ }^{(1)}$

Pour permettre à sa sœur de débuter ses études ,Mania accepte un métier sans prestige ainsi qu'une cruelle attente en résistant

« la clé de la maîtrise est cette habilité à surveiller nos pensées et nos émotions, à jongler entre la peur et le courage (....)̀̀ se recentrer lorsque nous sommes décalés.(...) la maîtrise de soi s'apprend, mais elle doit être mis en action, on doit la vivre pour qu'elle s'imprime dans tous les fibres de notre être.» $^{(2)}$

En se plaçant chez les B. une famille polonaise riche, Mania s'attendait à avoir des parents bénévoles, des enfants gentils, amusants cependant sa déception était fruste.

Les lettres de la petite institutrice à sa cousine Henriette nous faisaient estimer la différence de l'entourage qu'elle a quitté. Dans ce milieu bourgeois où l'a inséré son nouveau métier ,sa primauté éclate même à ses yeux. Mania «est fière de ses origines et de la formation qu'elle a reçue(...)[Elle] n'a pas seulement retiré, de sa première expérience, des enseignements philosophiques sur l'espèce humaine, et sur «les gens que la fortune a démoralisés.»» ${ }^{(3)}$

Mania ne gagnait pas assez d'argent et surtout elle en a dépensé trop : ses appointements éparpillés en de petits achats lui laissant,

1) http://www.messagerspirituel.com/laclede la maîtrisede soi.htm $18 / 10 / 2015$

2) Rish (M) http://www.messagerspirituel.com/lacledelamaitrisedesoi.htm 18/10/2015

3) Curie, (E) Madame Curie, opcit., PP.71-72

Vol. 48 
à la fin du mois ,des économies anodines pourtant elle se prépare à aider Bronia en tenant aussi compte de la retraite de son père. Le sacrifice emmène le sacrifice, elle a accepté une place d'institutrice bien payée chez Monsieur et Madame Z...à la campagne dans une province lointaine malgré la séparation durant des années des êtres chers en particulier son père ainsi que l'isolement total. Le premier janvier 1886, jour du voyage était une des dates pénibles de Mania lors de l'adieu de son père en répétant plusieurs fois son adresse. Ses nouveaux patrons $M$ et Mme Z... étaient d'excellents gens, des administrateurs exploitant une partie des terres des princes Czartoryski à cent km au nord de Varsovie.

Écrivant à sa cousine Henriette le 3 février 1886, Mania lui a raconté que cette famille avait trois fils à Varsovie ( un à l'université ,deux dans des pensionnats). À la maison, Bronka (18 ans ), Andzia (10 ans), Stas ( 3 ans ) et Marychna ,une petite fille de six mois. Quant à son rôle, elle a eu sept heures de travail par jour :quatre avec Andzia, une enfant obéissante mais gâtée, trois avec Bronka avec laquelle elle a noué des relations amicales.

Etant partie vers une maison de campagne, Mania attendait de voir des prairies des forêts mais le comique était «d'apercevoir une cheminée d'usine, haute, agressive et qui, bouchant et salissant le ciel, crache un panache opaque de fumée noire ?(...) il n'y a pas un taillis, pas un pré : rien que des betteraves(...) pâles et terreuses, empilées dans des chars à boufs, convergent lentement vers la fabrique, qui est une sucrerie. C'est pour l'usine que les paysans sèment, sarclent, récoltent.» ${ }^{(1)}$

Seule ,la petite institutrice écrivait beaucoup de lettres pour recevoir des réponses, des nouvelles de la ville de Varsovie . Pendant que s'écoulaient les semaines et les mois, Mania racontait à ses proches les imprévues de sa vie salariée, où les modestes tâches alternent avec les heures de compagnie ainsi que les divertissements obligatoires. Mais il y a eu en Mania un être

${ }^{1)}$ Curie, (E) Madame Curie, opcit., P.75

Vol. 48 
courageux et originel qui ne pouvait supporter longtemps cette vie routinière «l'idéaliste positive» était toujours présente, stimuler de se rendre profitable, de combattre.

«La maîtrise de soi est un processus qui se fait au jour le jour. La clé est d'être conscient de nos attitudes mentales. De faire l'inventaire de nos pensées et croyances qui eux influencent nos états émotionnels et par conséquent ce que l'on ressent en nous. Cela nécessite de se placer en observateur, de prendre de la distance, d'avoir une vision d'ensemble par rapport à soi-même, c'est l'art de se connaitre.» ${ }^{(1)}$

La plupart des enfants du village était illettrés, rares ceux qui allaient à l'école y ont appris l'alphabet russe. Comme C'était une bonne occasion de créer des cours clandestins de polonais, d'exciter ces jeunes cerveaux à la beauté de la langue et de l'histoire nationale.Et pour commencer ces cours,Mania a obtenu la permission du chef de famille et de sa femme $\mathrm{M}$ et Mme $\mathrm{Z}$ Quant à leur fille aînée Bronka, dans son regard «Mania lit l'ardeur et la résolution.» (2)

Écrivant à sa cousine Henriette, le 3 septembre 1886 pour lui déclarer son expérience «(...) Bronka et moi ,nous donnons pendant deux heures (...) des leçons à des enfants de paysans. (...) Ils travaillent avec beaucoup de bonne - volonté-pourtant notre tâche est parfois très difficile.» ${ }^{(3)}$

En décembre de la même année, Mania a de nouveau écrit à sa cousine pour lui communiquer les nouvelles de cette expérience audacieuse dont elle accueillait ses fruits «(...) le nombre de mes

1) Rish (M) http://www.messagerspirituel.com/lacledelamaitrisedesoi.htm 18/10/2015

2) Curie, (E) Madame Curie, opcit., P.79

${ }^{3)}$ Idem

Vol. 48 
élèves paysans atteint maintenant dix-huit. Naturellement,(...)ils me prennent deux heures par jour.Le mercredi et le samedi, je reste avec eux plus longtemps- jusqu'à cinq heures de suite.» ${ }^{(1)}$

Ces petits élèves fils et filles de servantes, de paysans ,d'ouvriers de la sucrerie ne pressentaient pas que « Mademoiselle Marya » a médité amèrement sur sa propre ignorance, ils ne savaient plus que le rêve de leur institutrice est de redevenir une élève et qu'elle désirait au lieu d'instruire ,apprendre.

\section{«( ... ) Toute notre vie consiste en une longue chaîne d'apprentissage d'où l'on apprend à devenir des êtres responsables, des maîtres dans l'art de maîtriser notre nature corporelle et mentale.» (2)}

Il était curieux que cette créature géniale souffre et se décourage tel une enfant de dix-neuf ans qu'elle était. Il était curieux aussi de la voir déduire et dans l'instant même où elle réclame avoir renoncé à tout, rivaliser avec une vaillance enragée contre l'ensevelissement.

$$
\begin{aligned}
& \text { «(...)Nous sommes certes influencées de toutes parts et } \\
& \text { interagissons avec notre environnement, mais il n'en tient qu'à } \\
& \text { nous de choisir la façon que l'on va réagir ou ne pas agir» }{ }^{3)}
\end{aligned}
$$

Éloignée dans une maison de campagne, Mania était privée d'orientations, de conseils. Elle tâtonnait par hasard, dans le labyrinthe des connaissances qu'elle désirait conquérir et que lui présentaient en somme, des manuels démodés. Mais avec une ténacité de paysanne, elle poursuivait son effort en affirmant

\footnotetext{
1) Idem

2) Rish (M) http://www.messagerspirituel.com/lacledelamaitrisedesoi.htm 18/10/2015

3) Rish (M) http://www.messagerspirituel.com/lacledelamaitrisedesoi.htm $18 / 10 / 2015$

Vol. 48 


\section{«(....)Mes études solitaires étaient hérissées de difficultés.L'éducation scientifique que j'avais reçue au lycée était très incomplète- bien inférieure au programme $d u$ baccalauréat en France. .J'essayais de la compléter à ma manière, à l'aide de livres réunis au petit bonheur.» ${ }^{(1)}$}

Racontant, en plus, une de ses journées de Szczuki dans une lettre à sa cousine Henriette en disant «.(...)l'étude suivie d'un seul sujet pourrait lasser ma précieuse

cervelle, déjà fort surmenée!Quand je me sens absolument inapte à lire utilement, je résouds des problèmes d'algèbre et trigonométrie,qui ne supportent pas de fautes d'inattention.... ${ }^{(2)}$

L'être humain est le maître absolu de ce qui se passe dans son esprit ,il est de son devoir de bien faire son choix de quoi on le nourrit, moment par moment, pour le réserver en bonne santé et le diriger pour que la vie soit positive en dépit des incidents négatifs de son environnement.

«La maîtrise de soi « concerne tout notre être :l'esprit, l'âme et le corps La maîtrise de notre esprit :nos pensées, notre volonté....." ${ }^{(3)}$

Petit à petit, l'adolescente Mania s'est modifiée en une fille charmante,sa peau et ses cheveux sont fascinants. Elle a de superbes poignets, des chevilles sveltes. Et son visage attire l'attention par ses yeux gris de cendre, profondément enfoncés sous les sourcils et que l'expression stupéfiante du regard fait paraître grands.

Revenu à Szczuki, le fils aîné de $\mathrm{M}$ et Mme Z...pour passer des jours de fête puis de longues vacances, a trouvé une gouvernante

\footnotetext{
${ }^{1)}$ Curie, (E) Madame Curie, opcit., P.82

2) Curie, (E) Madame Curie, opcit., P.83

3) http://www.pasteurweb.org/Etudes/le\%25Fruit\%2520de\%2501\%27Esprit/
} La patience.htm

Vol. 48 
spirituelle bien élevée, qui crée des vers à l'improviste aussi bien qu'elle monte à cheval,qui est totalement différente.

Il en est tombé amoureux et de même Mania s'est épris de ce beau et gentil étudiant. C'était donc sans crainte, sans hésitation que Casimir a demandé à ses parents s'ils approuvent ses fiançailles Mais la réponse ne s'est fait pas attendre. En un instant, dans une maison où l'on traitait Mania comme une amie, les barrières sociales se sont redressées, indépassables. Le fait qu'elle est cultivée, brillante, d'une réputation sans défavorable et que son père est digne de considération et d'estime à Varsovie.pourtant, rien ne compte devant cette phrase blessante «On n'épouse pas une gouvernante. ${ }^{(1)}$

Admonesté, apostrophé, houspillé, l'étudiant Casimir sentait fondre sa décision.Il a craint les remontrances ainsi que la fureur des siens. Et Mania, blessée par le mépris orgueilleux de ces êtres qui lui étaient inférieurs, s'est enfermée dans un silence agacé.

$\mathrm{Ne}$ pouvant quitter les $\mathrm{Z} \ldots$ de peur d'inquiéter son père. Particulièrement, elle ne saurait s'offrir le luxe de renoncer à une si meilleure place C'était Mania, qui, avec M.Sklodowski a payé les études de son aînée Bronia à la Faculté de Médecine en lui envoyant une quinzaine de roubles parfois vingt presque la moitié de ses gages.

Entre les Z...et Mania, il n'y a eu aucune explication directe, aucun débat embarrassant . Mieux vaut donc boire le camouflet et rester à Szczuki, comme si rien ne s'était arrivé.

«La maîtrise de soi implique de s'apprivoiser et non de se flageller si on réagit trop fortement envers une situation ou envers une personne. L'important est de retrouver notre équilibre» ${ }^{(2)}$

1) Curie, (E) Madame Curie, opcit., P.85

2) Rish (M) ,http :www.messagerspirituel.com/lacledelamaitrise de soi.htm $18 / 10 / 2015$

Vol. 48 
Il est clair que les êtres humains trouvent dans leur chemin de vie certaines personnes qui tentent souvent de les déstabiliser. Et si elles y réussissent ils seront toujours les grands perdants.

"Par contre, si vous réussirez à incorporer le fait que vous valez mieux que cela, ce genre de tentatives n'aura pour seul effet que de démontrer la puérilité de ses auteurs.» ${ }^{(1)}$

Mania a traversé des semaines pénibles, elle a eu des jours très durs mais la seule chose qui apaisait leur souvenir c'était que, malgré tout, elle est sortie de cet état honnêtement, la tête haute.

$$
\begin{aligned}
& \text { "(..)douter de vous-même ébranle votre } \\
& \text { crédibilité aux yeux du monde et par conséquent } \\
& \text { affaiblit la maîtrise de soi.» }{ }^{2)}
\end{aligned}
$$

Dans la lettre écrite à Henriette le 10 décembre $1887 .$. , Mania a parlé de ses projets pour l'avenir,ces projets étaient tellement modestes : son rêve a été de trouver un coin à elle pour pouvoir habiter avec son père qui lui manquait beaucoup. Et elle, pour ressentir l'indépendance,une maison, elle va donner la moitié de sa vie.

Si donc la chose paraît possible, elle laisserai Szczuki-ce qui ne peut pourtant se réaliser avant quelque temps-pour aller s'installer à Varsovie en prenant une place de professeur dans un pensionnant et en gagnant le complément avec des leçons.

$$
\begin{aligned}
& \text { «(...) Nos pensées et nos émotions ne sont pas notre } \\
& \text { essence profonde, elles sont des outils qui nous servent }
\end{aligned}
$$

\footnotetext{
1) Jean-Baptiste , http ://www.reussitepersonnelle.com/maitise -de -soi/ $18 / 10 / 2015$

2) Jean-Baptiste , http ://www.reussitepersonnelle.com/maitise -de -soi/ $18 / 10 / 2015$

Vol. 48 
comme bâtisseur de notre vie, tout comme la vue nous sert à voir notre environnement extérieur.» (1)

Trois années ennuyeuses se sont filées depuis que «Mademoiselle Marya» était institutrice : beaucoup de travail, pas d'argent, quelques petites joies, une affliction. Certains événements infimes, en apparence ont changé les jeux de la partie discrète où se jouait le destin de Mania.La première chose qu' a fait Bronia était de commander à Mania de ne plus lui envoyer d'argent. La seconde était de demander à son père de conserver sur les quarante roubles qu'il lui a donné chaque mois, huit roubles voués à rendre petit à petit les sommes qu'elle a prises de sa cadette. Dès ce moment, la fortune de Mania a débuté de zéro à augmenter.

À Szczuki, en 1889, la tâche de Mania chez les Z...a touché à sa fin. Il fallait trouver une autre place.La jeune institutrice en a eu une en vue, chez des grands industriels qui habitaient Varsovie, les F...C'était enfin un changement fort intense. Mlle Sklodowska a pris congé des Z...libérée, elle a respiré l'air de sa ville natale et dans le train, elle est allée rejoindre sur une maussade plage de la Baltique, à Zoppot, ses nouveaux patrons. Mme F....était très belle, très coquette, très riche. Elle avait des fourrures, des bijoux et «des robes de worth » dans ses placards. Mania a connu, en spectatrice, les choses futiles et fascinantes que la fortune pouvait offrir à une femme gâtée .Première et dernière rencontre avec le luxe ! Elle a été rendue accueillante par la bonté grâce de Mme F....qui, elle aussi, séduite par «l'exquise Mademoiselle Sklodowska» a chanté les éloges de Mania.

1) Rish,(M) , http.//www.messager spirituel.com /lacledelamaitrise de soi. htm 18/10/2015 
Et Soudain, une pauvre lettre de Paris, en mars 1990 que Bronia a écrite entre deux séances à l'amphithéâtre où la magnanime fille offre à Mania pour l'année prochaine l'hospitalité dans sa nouvelle demeure où elle trouvera le gîte et la nourriture mais c'est à Mania d'avoir quelques centaines de roubles pour venir à Paris ainsi que pour les inscriptions à la Sorbonne

«Croit-on que Mania, enthousiasmée, va
répondre qu' elle exulte de bonheur,qu'elle
arrive?Pas du tout!(...)Le démon du
sacrifice la rendrait capable de manquer,
exprès, sa destinée. Parce qu' elle a promis à
son père d'habiter avec lui, parce qu' elle veut
aider sa sœur Hela ,aider son frère Joseph,
Mania ne veut plus partir!» ${ }^{(1)}$

Définitivement, il est décidé qu'après avoir rempli son engagement chez Mme F..., Mania va rester pendant un an à Varsovie auprès de son père, déchargé récemment de son poste, elle va augmenter ses économies en donnant des leçons. Et ensuite, elle partira...

Dans son pays natal, Mania a retrouvé l'ambiance qui lui était chère : un foyer à soi, la présence de son père âgée monsieur Sklodowski, des dialogues intéressantes qui ont excité l' esprit. En outre, l'université lui a ouvert ses portes occultes: enthousiasme inégalable, événement primordial: pour la première fois, Mania s'enfonce dans un laboratoire, son cousin, Joseph Boguski, y a dirigé ce qui était majestueusement nommé Le Musée de L'Industrie et de L'Agriculture. Cette appellation exprès hautaine, était une façade réservée aux autorités russes. Rien n'a entravé, derrière les vitres de ce musée d'enseigner les sciences à des Polonais.

1) Curie, (E) Madame Curie, opcit., P.97

Vol. 48 
«J'avais peu de temps pour travailler dans ce laboratoire (...) Je ne pouvais généralement y aller que le soir après dîner ou le dimanche (....)J'essayais de reproduire diverses expériences décrites dans les traités de physique ou de chimie, et les résultats étaient parfois inattendus. De temps en temps, une petite réussite inespérée venait m'encourager, (....) Dans l'ensemble(...) le progrès en ces matières n'est ni rapide ni aisé, je développai, au cours de ces premiers essais, mon goût de la recherche expérimentale» ${ }^{(1)}$

De même, Mania n'a pas nié que d'autres fois elle succombait dans le désespoir à cause d'accidents ou d'échecs issus de son inexpérience.

\section{«(...)Il n'y a pourtant aucune honte à ne pas tolérer l'échec ou à être susceptible. Le tout est d'en prendre conscience afin de ne pas exploser au moment où ces sentiments feront surface. C'est cela, la maitrise de soi.» ${ }^{(2)}$}

Rentrée chez elle tard, ayant laissée à regret les électromètres, les tubes à essais, les balances de précision, Mania s'est déshabillée et s'est étendue sur un étroit divan. Mais elle ne pouvait dormir.Une excitation différente de toutes celles qu'elle a ressenties l'a retenue, frémissante, hors du sommeil. Sa vocation, longtemps indéterminée, a surgi, la somme de céder à un ordre clandestin.

La jeune fille était hâtée, torturée. En tenant dans ses mains agiles les éprouvettes du Musée de l'industrie et de l'Agriculture, Mania, prodigieusement, a rattrapé les souvenirs de son enfance,

1) Curie, (E) Madame Curie, opcit., PP.99-100

2) Jean- Baptiste, http://www.reussitepersonnelle.com/maitrise-de-soi/ $18 / 10 / 2015$

Vol. 48 
les appareils de physique de son père avec lesquels, autrefois, elle avait envie de jouer. Elle s'est pris de nouveau le fil de sa vie.

«Pour développer une psychologie
équilibrée, il faut être capable de se
soumettre à l'introspection. En analysant
chaque compartiment de notre vie, vous
mettez bien en lumière les domaines ou
votre capacité à contrôler vos émotions
vous fait défaut.» 1 (1)

Une lettre écrite de Varsovie par Mania à sa sœur Bronia dans laquelle elle a demandé une réponse définitive si vraiment elle pouvait la prendre chez elle car elle peut venir et elle a de quoi payer ses dépenses.

$$
\begin{aligned}
& \text { "(...)Ce serait un grand bonheur, car } \\
& \text { moralement cela me remettrait d'aplomb, } \\
& \text { après les cruelles épreuves que j'ai } \\
& \text { traversées cet été, et qui influeront sur toute } \\
& \text { ma vie-mais d'autre part je ne veux pas } \\
& \text { m'imposer à toi» (2) }
\end{aligned}
$$

\section{Voyage de Marya à Paris}

Si Mania ne s'est pas lancée dans le premier train, c'est qu'il lui implique tout d'abord, avec parcimonieuse épargne, aménager le grand départ .Elle a mis sur une table tous les roubles qu'elle possède et auquels son père, a augmenté une modeste somme, pour lui très nécessaire. Et elle a commencé ses comptes sans oublier les conseils de Bronia

1) Jean- Baptiste, http://www.reussitepersonnelle.com/maitrise-de-soi/ $18 / 10 / 2015$

${ }^{2)}$ Curie, (E) Madame Curie, opcit., P102

Vol. 48 
«(....) emporter de chez soi ce qui est nécessaire à la vie, afin de n'avoir à Paris aucune dépense imprévue.(....) son linge, fait d'une toile forte, ses vêtements, ses souliers, ses deux chapeaux(....) une grosse malle de bois, brune et bombée, très rustique mais très solide et sur laquelle, amoureusement la jeune fille a fait peindre en larges lettres noires initiales: M.S. !» ${ }^{(1)}$

Il ne reste à la voyageuse que de la nourriture et de la boisson pour trois jours de train, le pliant pour le wagon allemand de la quatrième classe, des livres, un sac de caramels et une couverture. Mania a rêvé à ce voyage féerique si longuement attendu. Elle a tenté d'imaginer l'avenir. Si loin d'elle, la pensée qu'en grimpant dans le train, elle a enfin choisi entre «l'obscurité et le flambeau, entre la petitesse des jours égaux et une vie immense.» ${ }^{(2)}$

Au moment, où, ternie par l'accablant voyage, Mania a mis à terre du train sur le quai de la gare du Nord, le poids de la servitude s'est relâché soudainement, les épaules se sont réjouies, les poumons et le cœur se sont ressentis à l'aise. C'était pour la première fois que Mania respire l'air d'un pays libre.Et,dans son exaltation,tout lui semble merveilleux que les libraires vendent sans cœrcition les ouvrages du monde entier. Merveilleuses surtout «ces avenues droites inclinées en pente douce vers le cour de la ville[qui] la conduisent, elle, Mania Sklodowska, aux portes grandes ouvertes d'une Université.(...)La plus fameuse, celle qu'il y a des siècles on décrivait déjà comme un «abrégé de l'Univers», celle-là même dont Luther disait : C'est à Paris que se trouve la plus célèbre et la plus excellente école : on l'appelle la Sorbonne! ${ }^{(3)}$

Les cours changent d'une salle à une autre, des laboratoires précaires ont dû être placés dans des vieilles maisons de la rue

1) Ibid., P.103

${ }^{2)}$ Curie, (E) Madame Curie, opcit., P.104

3) Ibid., P.108

Vol. 48 
Saint-Jacques. Avec la petite somme d'argent qu'elle a massé, la jeune fille enfin a captivé le droit d'écouter les multiples leçons qu'il lui convient de choisir.Elle a eu sa place dans les « salles de manipulations» où orientée, conseillée, elle a pu manier des appareils, réussir des expériences simples, elle est actuellement une étudiante à la faculté des sciences.

Des obstacles inattendus se sont élevés devant elle dès ces premières semaines. Des phrases françaises entières prononcées d'une façon trop rapide. Elle penserait posséder une culture scientifique satisfaisante pour suivre facilement ses leçons Mais les études indépendantes à la campagne, les connaissances reçues envoyées avec M.Sklodowski, les expériences essayées au Musée de l'industrie et de l'Agriculture ne pourraient remplacer le vigoureux baccalauréat des lycéens de Paris.Dans l'amphithéâtre, Marie a trié une place près de la chaire. Elle a posé d'une façon méthodique son porte -plume ainsi que son cahier d'où elle écrira des notes.

«(...) un élan qui ne peut se comparer qu'à l'amour jaillit de l'âme de la jeune fille vers l'infini $d u$ savoir, vers les choses et vers leurs lois.» ${ }^{(1)}$

Marie s'est lancée avec ardeur vers ce qui donnerait sa nouvelle existence.Elle décèle ainsi les joies de la camaraderie, de l'entraide que crée le travail à l'université.Mais sa grande timidité l'empêche d'avoir des liens avec des Français, elle se réfugie parmi ses compatriotes: deux mathématiciennes, le docteur Motz, le biologiste Danysz, Stanislas Szalay et le jeune Wojciechowski qui deviennent ses amis constituant au quartier latin une petite île de Pologne libre.

$$
\begin{aligned}
& \text { «(...) malgré l'exil, l'éloignement, ni Marie } \\
& \text { ni sa sœur n'ont quitté Varsovie.(...)Leur }
\end{aligned}
$$

1) Curie, (E) Madame Curie, opcit., P.111

Vol. 48 
patrie est avec elles, les tenant par mille attaches dont la moins forte n'est pas leur correspondance avec leur père .» ${ }^{(1)}$

La vie dans la rue d 'Allemagne est agréable et douce.Mais Marie n'y trouve pas le recueillement : Ne pouvant interdire à Casimir de jouer du piano ni de recevoir ses amis ni d'entrer dans sa chambre pendant qu'elle est en train de résoudre des équations compliquées de même ne pouvant écarter les clients des jeunes médecins de leur irruption dans le logement. En plus, le trajet de la Villette à la Sorbonne prend une heure et le prix de deux omnibus est si cher. Marie décide de vivre au quartier latin près de l'université, des laboratoires et des bibliothèques.
«(....) la qualité de notre vie est déterminée par la qualité de nos pensées, qui eux déterminent nos choix et par conséquent ce que l'on fait de notre vie. (....) Toute pensée est une image qui produit la toile de notre réalité présente.» ${ }^{(2)}$

Entre Marie et Casimir est née une affinité fraternelle qui subsiste autant que leur vie et entre Mania et Bronia a lieu depuis des années un impressionnant roman : le roman du sacrifice et du dévouement.

Dès que Marie s'est privée de sa propre volonté du logis et de la nourriture qu'elle trouvait chez les Dluski, elle pourvoit seule à toutes ses dépenses. Et sa pension s'est faite de ses économies partagées en tranches et les quarantes roubles que peut lui envoyer son père par mois ? Tel est le problème le plus pressant à résoudre mais il n'est guère arrivé que Marie ne trouva pas la solution d'un problème.

\footnotetext{
1) Ibid., PP.115-116

2) Rish,(M) http://www.messagerspirituel.com/L'art de diriger notre Esprit.htm 9/7/2016
} 


$$
\begin{aligned}
& \text { «(...) Toute chose impossible peut devenir possible, } \\
& \text { selon la mesure de nos pensées et effort que l'on y } \\
& \text { met.» } 1 \text { (1) }
\end{aligned}
$$

Donc, il faut garder les yeux fixés sur ce qu'on veut vraiment, se concentrer de suite sur ce qu'on aimera et s'élancera dans cette voie. Marie diffère de ses collègues polonaises :les unes occupant à trois ou quatre le même logis prenant leurs repas en commun tandis que les autres qui habitent seules vouent plusieurs heures journellement à faire le ménage, la cuisine, à raccomoder et à force d'adresse, mangent à leur appetit, se chauffent et s'habillent telle est la méthode ajustée autrefois par Bronia. Mania insiste trop à son calme, sa sérénité pour partager une demeure avec des amies .Elle est trop obsédée par le travail que pour son confort.

\section{«(....)elle a supprimé de son programme les distractions, les réunions amicales, le contact avec les humains. De même décide-t-elle que la vie matérielle n'a aucune importance (....)Forte de ce principe, elle se compose une existence de spartiate, étrange, inhumaine.» ${ }^{(2)}$}

Les chambres que loue Marie ayant de la ressemblance par leur modestie et par leur inconfort: la première est placée dans un pauvre hôtel. Après avoir cherché le calme absolu, la jeune fille loge une mansarde pareille aux chambres de domestiques où l'on trouve un réduit infime prenant la lumière par une lucarne qui donne immédiatement sur le toit en pente. Par le moyen de cette fenêtre, se révèle un carré de ciel. Ni éclairage, ni chauffage, ni eau. Mania décore ce bâtiment de tous les objets apportés de Pologne.

1) Rish,(M) http://www.messagerspirituel.com/L'art de diriger notre Esprit.htm 9/7/2016

2) Curie, (E) Madame Curie, opcit., P.121

Vol. 48 
«(....)Il faut savoir être l'observateur et l'acteur de notre vie, car la vie est interaction. C'est ce fin équilibre entre être et faire» ${ }^{(1)}$

Avec ses quarante roubles, elle est arrivée à vivre et en se dépouillant de l'essentiel, à s'offrir quelquefois certain luxe : une soirée au théâtre, une promenade en banlieue d'où elle revient en rapportant des fleurs. Pour la plupart du temps, Marie va à pied à la Sorbonne. Le minimum de charbon: un ou deux sacs de «boulets» chaque hiver qu'elle achète et qu'elle fait lever ellemême au sixième étage en prenant une pause à chaque palier afin de reprendre une haleine. En ce qui concerne l'éclairage: quand il fait nuit, l'étudiante se retire dans ce refuge élu qui se nomme la bibliothèque Sainte- Geneviève où le gaz est allumé. Assise à une des grandes tables rectangulaires, une Polonaise pauvre peut étudier jusqu' à dix heures du soir, l'heure de la fermeture .Il suffit de suite d'avoir assez de pétrole pour illuminer chez elle jusqu'à deux heures du matin.Travaillant ainsi dans son propre logis, il lui paraît que son destin encore minuscule rejoint discrètement les hautes existences ainsi que les grands savants du passé qu'elle admire tant et plus.

$$
\begin{aligned}
& \text { "comme elle enfermés dans leurs cellules mal } \\
& \text { éclairées, comme elle détachés du siècle, } \\
& \text { comme elle éperonnant leur esprit pour le } \\
& \text { porter au-delà de la connaissance acquise.» }
\end{aligned}
$$

N'en déduisons pas que Marie, dans un accès d'élégance, achète quelquefois à bas prix un coupon de tissu et se taille un vêtement neuf. Elle paraît avoir jurer, au contraire, de ne jamais laisser ses robes usées de Varsovie, elle les porte tout en les nettoyant et les

\footnotetext{
1) Rish, (M), http://www.messagerspirituel.com/lacledelamaitrisedesoi.htm 18/102015

2) Curie, (E) Madame Curie, opcit., P.132
}

Vol. 48 
ravaudant. Elle cède encore à faire la lessive dans sa cuvette lorsqu'elle a besoin de repos.

$$
\begin{aligned}
& \text { "(..) elle ne mange que du pain beurré, en } \\
& \text { buvant du thé. Lorsqu'elle a envie d' un festin, } \\
& \text { elle pénètre dans une crémerie (...)où on lui sert } \\
& \text { deux oufs, ou bien elle achète un morceau de } \\
& \text { chocolat, un fruit.» } 1 \text { (1) }
\end{aligned}
$$

\section{Rêve humanitaire et Rêve scientifique}

Complètement plongée dans les études, Marie poursuit des cours de mathématiques de physique, de chimie. La méthode manuelle des épreuves scientifiques lui devient peu à peu usuelle, ordinaire ce qui lui donne la gaieté de se voir confier par le professeur Lippmann des recherches peu importantes, mais qui lui donneront l'occasion de manifester sa dextérité et l'originalité de son esprit.

Au laboratoire de physique de la Sorbonne, Marie se prend à aimer avec fureur cette ambiance de concentration et de silence que, jusqu'à son dernier souffle, elle privilégiera à tout autre. Ces quatre années d'études n'étaient ni les plus heureuses ni les plus impeccables mais les plus proches des cimes de la mission humaine un zèle immense donne à l'éternelle étudiante Polonaise le pouvoir d'ignorer les pénuries, de magnifier sa vie vile. Marie décide de passer deux licences celle de physique et celle de mathématiques. Ses projets, autrefois si modestes, se gonflent rapidement qu' elle n'a ni le temps ni surtout l'audace de les confier à son père M.Sklodowski.

1) Curie, (E) Madame Curie, opcit., P.123 Vol. 48 
Certaine que Marie est vouée à un grand avenir ,Mlle Dydynska a secoué ciel et terre pour lui faire attribuer la «bourse Alexandrowitch » destinée aux étudiants de mérite voulant continuer leur effort à l'étranger.

Avec une ladrerie passionnée, Marie tente d'étirer les six cents roubles,de demeurer longuement dans l'éden des amphithéâtres et des laboratoires. Plusieurs années plus tard, avec la même ladrerie passionnée, elle épargnera six cent roubles

«sur son premier gain - une étude technique
qui lui sera commandée par la Société
d'Encouragement pour l'Industrie
Nationale- et elle ira les porter au
secrétaire de la Fondation Alexandrowitch,
stupéfait d'une restitution sans précédents
dans les annales du comité.» 1 (1)

A L'université que les Goncourt appelaient la « mère nourricière de l'étude» Marie discute avec Paul Painlevé, avec Jean Perrain et avec Charles Maurin, de futurs maîtres de la Science Française, n'ayant pas le temps de donner à l'amitié ou à l'amour.
«(....)Elle est soutenue par une volonté d'airain, par un goủt maniaque de le perfection, par un entêtement incroyable. Systématiquement, patiemment, elle atteint chacun de ses buts : elle est reçue première à la «Licence ès Sciences Physique» en1893, seconde à la "Licence ès Sciences Mathématiques $_{\text {en } 1894 . »{ }^{(2)}}$

\footnotetext{
1) Curie, (E) Madame Curie, opcit., PP.130-131

2) Ibid., P.126
}

Vol. 48 


\section{Rencontre de Pierre Curie}

Un polonais, M. Joseph Kowalski, professeur de physique à l'université de Fribourg réside en France avec sa femme, dont Marie a autrefois fait la connaissance à Szczuki. M.Kowalski donne des conférences et assiste aussi aux séances de la société de Physique à Paris. Depuis son arrivée, il s'est enquis de Marie en lui demandant ses nouvelles. L'étudiante lui a confié ses soucis : La Société pour l'Encouragement de l'Industrie Nationale lui a commandé une étude sur les propriétés magnétiques de divers aciers. Elle a débuté ses recherches au laboratoire du professeur Lippmann. Mais il lui faut analyser des minerais ,rassembler des échantillons de métaux. Ceci exige une installation trop encombrante pour un laboratoire déjà surchargé. Et Marie ne sait que faire? dans quel édifice réaliser ses expériences?

Après quelques instants de réflexion, M.Kowalski lui répond qu'il connaît un savant de grande valeur travaillant à l'Ecole de physique et de chimie rue Lhomond peut-être disposerait-il un bâtiment disponible? Pendant une calme soirée passée dans une chambre de la tranquille pension de famille qu'habite le couple $\mathrm{M}$ et Mme Kowalski, une attirance immédiate rapproche le physicien Français Pierre Curie de la physicienne polonaise.

Pierre Curie est le second fils d'un médecin, le docteur EugèneCurie, lui- même fils d'un médecin . Etant d'origine alsacienne et protestante, les Curie, jadis des bourgeois modestes sont devenus des intellectuels, des savants. Les deux jeunes fils du docteur, Jacques et Pierre ont été, dès leur enfance, attirés par les sciences. Ils proclament la découverte d'un phénomène primordial la «piézoélectricité», et leur effort expérimental les amène à inventer un appareil nouveau dont les applications sont 
multiples : le quartz piézoélectricité servant à mesurer d'une manière précise de faibles quantités d'électricité.

En1883, les deux frères se séparent: Jacques est nommé professeur à Montpellier, Pierre devient chef de travaux à $1^{\prime}$ Ecole de physique et de chimie de la ville de Paris. Malgré que les manipulations des élèves de $1^{\prime}$ Ecole lui prennent énormément de temps, il poursuit des travaux théoriques sur la physique cristalline.Ces travaux conduisent à l'énoncé du Principe de Symétrie, qui deviendra une des assises de la science moderne. Continuant ses études expérimentales, Pierre Curie invente et fabrique une balance scientifique ultra-sensible, la «balance Curie » puis il entreprend des quêtes sur le magnétisme et obtient un but capital: la découverte d'une loi essentielle, la « loi de Curie ».

Pierre Curie a été charmé par Marie Sklodowska . Avec une délicate tenacité, il tente de se rapprocher de la jeune Polonaise .Il l'a revue presque deux ou trois fois aux séances de la Société de Physique où elle prêtait l'oreille aux communications des savants sur des études nouvelles.

Il 1 'a vue au laboratoire du professeur Lippmann dans son sarrau de toile, courbée, tranquille, sur ses appareils. Et puis, il a demandé de lui rendre visite. Bienveillante, réservée, elle $1^{\prime}$ a reçu dans sa petite chambre et Pierre, ayant le cœur serré, a apprécié pourtant au plus profond de lui- même la finesse harmonie du personnage et du décor. L'intimité entre les deux jeunes physiciens se développe à mesure que multiplient le respect et l'admiration réciproques, la confiance. Pierre Curie est déjà l'interné de l'intelligente Polonaise, il lui obéit en écoutant ses avis.

Au cours de la deuxième visite de Pierre Curie chez Marie au milieu de quelques marguerites blanches rapportées d'une 
promenade faites par les deux ensemble, le physicien commence à parler de sa vie avec son père, sa mère dans un petit pavillon à Sceaux évoquant son enfance lunatique, ses promenades interminables dans le bois avec son frère Jacques.

Marie l'écoute, étonnée " Que d'analogies, de correspondancesmystérieuses! Il suffirait de changer quelques détails ,de transporter le pavillon de Sceaux dans une rue de Varsovie pour que la famille Curie devînt la famille Sklodowski. » ${ }^{(1)}$

Pierre Curie a conversé maintes fois du futur. Il a demandé à Marie de devenir sa femme Mais cela n'a pas été réjoui. Epouser un Français signifie pour elle abandonner à jamais sa famille, de quitter son activité patriotique, négliger la Pologne, toutes ces choses se révèlent à Mlle Sklodowska comme autant de traîtrises effroyables. Elle ne doit pas! Elle ne peut plus supporter.

En se séparant du jeune savant découragé, lui offrant une amitié dont il ne lui suffit pas, Marie prend le train pour Varsovie sans donner sa parole. À Ce moment, de loin, il persiste de défendre sa cause par des lettres dessinées d'une écriture empruntée, un peu puérile essayant de le convaincre.

Après le retour de Marie à Paris , P. Curie recommence sa tendre défense. A sa manière, il est aussi obstiné qu'elle. Devant l'ancienne institutrice méprisée autrefois par une famille de nobles polonais cet homme exceptionnel se fait modeste, suppliant. Ayant appris que la jeune physicienne a parlé de lui aux Dluski, Pierre tente de sa part une nouvelle attaque. Il gagne complètement Bronia, qu'il a déjà rencontrée maintes fois à sa cause. En vraie «intellectuelle » slave, Marie est surmenée de

1) Curie, (E) Madame Curie, opcit., P.144 Vol. 48 
théories sur l'existence ainsi que ses devoirs. Plusieurs de ces théories sont charitables et belles, d'autres ne sont qu'enfantines

"(...) Le savant fait bon marché des
principes que Marie partage avec
quelques milliers de ses compatriotes
cultivées. Ce qui le retient et le fascine,
c'est son dévouement total au travail,
c'est son génie qu'il pressent, c'est aussi
son courage et sa noblesse. Cette fille
gracieuse a le caractère et les dons d'un
grand homme.»

\section{Mariage de Marie et de Pierre}

Le 14 juillet 1895, Joseph, le frère de Marie lui envoie de Varsovie la chaleureuse rémission de la famille Sklodowski exprimée dans des phrases très sensibles.

"(...) je suis persuadé que de toute ton âme
tu resteras toujours une Polonaise (...) Je
préfère cent fois te savoir à Paris,
heureuse et contente, plutọt que de te voir
revenir dans notre pays, brisée par le
sacrifice d'une vie entière, et victime
d'une conception trop subtile de ton
devoir.» (2)

Enfin, le 26 juillet, à cette date importante, Mlle Sklodowska devient Madame Pierre Curie. Marie adore l'idée de ce mariage qui, jusque les minimes détails de la grande journée, sera très différente de tous les autres mariages. Pas de robe blanche, pas d'anneau d'or, pas de repas de noces.

\footnotetext{
1) Curie, (E) Madame Curie, opcit., P.151

2) Ibid., P.152

Vol. 48 


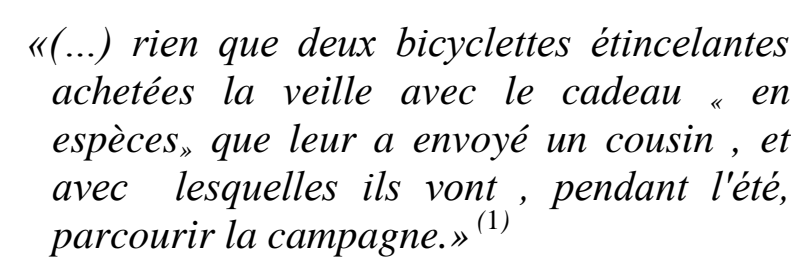

Repris par l'obsession de son travail, Pierre a oublié brusquement les bois, le ciel, la flore et la faune de l'étang assoupi .Il pense aux énormes difficultés de ses recherches, aux énigmes troublantes de la croissance des cristaux en entendant la tendre voix de Marie les questions perspicaces et les réponses raisonnées .

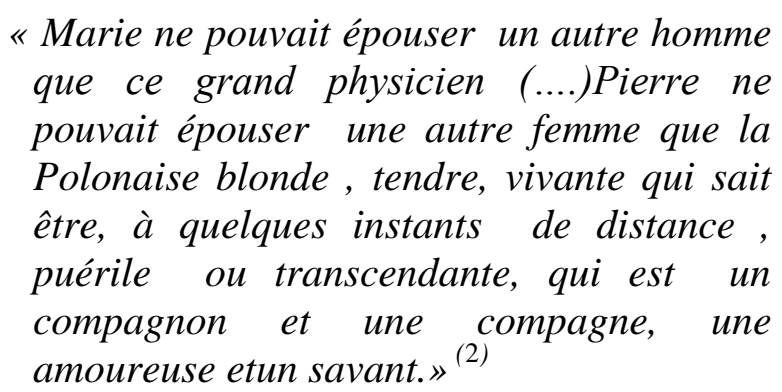

La vie de Pierre est tendue vers un but : faire des recherches scientifiques auprès de son épouse bien-aimée qui, elle aussi, vit pour la recherche scientifique Quant à la vie de Marie est plus difficile encore parce qu'à l'obsession du travail s'ajoutent les modestes et fatiguantes besognes des femmes. Le difficile est d'effectuer en vingt- quatre heures l'accablante besogne d'une journée or Marie passe la plupart de son temps au laboratoire de l'école où on lui a arrangé une place.

«Le Laboratoire, c'est le bonheur !» ${ }^{(3)}$

${ }^{1)}$ Curie, (E) Madame Curie, opcit., PP.153-154

2) Ibid., P.158

3) Ibid., P.161

Vol. 48 
Marie est reçue première au concours d'agrégation de l'Enseignement Secondaire. Engagés, Pierre et Marie ont gagné la rue Glacière et au même moment ils ont gonflé les pneus de leurs bicyclettes, rempli leurs sacs en se dirigeant vers l'Auvergne : voyage d'exploration.

Ecrivant à sa chère Kazia le 2 mars 1897 de ses nouvelles en disant qu'elle va avoir un enfant.

Depuis plus de deux mois, elle a des étourdissements incessants toute la journée du matin au soir. Le 12 Septembre, Marie donne naissance à une belle fille, Irène, un futur prix Nobel ! Le docteur Curie assiste à l'accouchement que Mme Curie supporte en ayant les dents serrées sans aucun cri. L'idée de choisir entre la vie de famille et la carrière scientifique ne passe même pas à l'esprit de Marie. Elle a décidé de «mener de front» l'amour, les maternités, la science et à ne jamais duper. Passion et résolution, elle y réussit.

$$
\begin{aligned}
& \text { «Être maître de soi est un savoir- faire, qui } \\
& \text { s'apprend et s'expérimente toute notre vie } \\
& \text { durant(.....) un art de vivre perpétuel et infini qui } \\
& \text { se perfectionne et qui s'améliore jusqu'à notre } \\
& \text { dernier souffle.» }{ }^{(1)}
\end{aligned}
$$

Sur l'ordre du médecin, Marie doit abandonner d'allaiter sa fille ce qui l'obligera à prendre une nourrice malgré le chagrin que cela le fera ainsi que la dépense. Mais matin, midi, soir et nuit c'est elle qui la change, la baigne et l'habille. La nourrice promène l'enfant au parc Mont Souris, tandis que la jeune mère s'active devant ses appareils de laboratoire et note le travail sur

1) Risk (M) http://www.messagerspirituel.com/lacledelamaitrisedesoi.htm $18 / 10 / 2015$

Vol. 48 
les aimants qui sortira au bulletin de la société pour l'Encouragement de l'Industrie Nationale.

La même année, à trois mois d'interstice, Marie met au monde sa première fille et le résultat de ses premiers essais.

$$
\begin{aligned}
& \text { «uel chemin parcouru, depuis le matin de } \\
& \text { novembrel891 où une Polonaise encombrée de } \\
& \text { paquets arrivait à la gare du Nord, (....) Elle a } \\
& \text { vaincu des obstacles humbles et gigantesques, sans } \\
& \text { se douter un instant de l'appel qu'elle faisait à une } \\
& \text { ténacité, à un courage exceptionnels.» } 1 \text {. }
\end{aligned}
$$

Deux licences, le concours d'agrégation, une étude sur l'aimantation des aciers trempés tel est l'inventaire de l'activité de Marie à la fin de 1897. La phase suivante, dans l'évolution logique de sa carrière, c'est le doctorat qui exige de choisir un thème de recherches fournissant une matière féconde, inédite. Marie porte en elle, depuis son enfance, la curiosité et l'intrépidité des explorateurs. Ces deux facteurs qui l'ont poussée, autrefois, à quitter son cher pays Varsovie pour découvrir Paris et la Sorbonne, qui lui a fait choisir une chambre solitaire du quartier latin au mœlleux appartement des Dluski.

Marie fouillant les travaux de physique les plus modernes, les comptes rendus des dernières études expérimentales, s'empare des travaux du physicien Français Henri Becquerel. Elle les lit, les relit et les analyse avec sa prudence habituelle. Àprès la découverte des rayons X par Roetgen, Henri Poincaré avait eu la notion de chercher et de rechercher si des rayons pareils aux rayons X n'étaient pas émis par les corps «Fluorescents», sous l'effet de la lumière.

1) Curie, (E) Madame Curie, opcit., PP.168-169 
Entrainé par le même problème, Henri Becquerel avait scruté les sels d'un «métal rare», l'urane Mais au lieu de trouver le phénomène prévu, il en avait contemplé un autre, tout hétérogène, inexplicable: les sels d'urane émettaient d'une manière spontanée, sans action préalable de la lumière, des rayons d'une nature étrangère. Un composé d'urane, posé sur une plaque photographique cerclée de papier noir, impressionnait celle-ci à travers le papier. Et, semblables aux rayons $\mathrm{X}$, ces étonnants rayons « uraniques» déchargeaient un électroscope en rendant conducteur l'air ambiant.

Henri Becquerel s'était confirmé que ces propriétés ne relevaient pas d'une insolation préliminaire et qu'elles duraient lorsque le composé d'urane était retenu longuement dans l'obscurité. Il avait découvert le phénomène auquel Marie devait nommer le nom de radioactivité. Mais l'origine de ce rayonnement restait un mystère.

Comme point de départ, il existe les informations présentées par Henri Becquerel à l'Académie des sciences au cours de l'année 1896. Cela va être intéressant de se jeter dans l'aventure, dans un domaine inexploré. Il ne reste plus qu'à trouver une salle où Marie puisse exécuter ses expériences. Plusieurs marches de Pierre auprès du directeur de l'École de Physique aboutissent à un modeste résultat: la libre disposition est cédée à Marie d'un atelier vitré situant au rez-de-chaussée des bâtiments de l'École. Privée d'une installation électrique convenable et de tout ce qui forme le matériel de départ des recherches scientifiques, Marie trouve le moyen de faire actionner ses appareils dans ce local or le climat de cette petite salle, fatal aux électromètres, n'est pas excellent pour la santé de Marie..... Ayant trop froid, la physicienne se venge en marquant dans son carnet de travail les degrés centigrades que désigne le thermomètre ce qui l'amène à écrire plus tard « Nous n'avions pas d'argent, pas de laboratoire et pas 
d'aide pour mener à bien cette tâche importante et difficile(....) C'était comme de créer quelque chose avec rien....» (1)

Dans un pareil lieu, un ouvrier refusait de travailler cependant Marie et Pierre s'y résignent.

$$
\begin{aligned}
& \text { "(...) Les ressentis négatifs qui stagnent au fond } \\
& \text { de vous et qui sont stimulés par votre } \\
& \text { environnement ne doivent pas devenir des } \\
& \text { gâchettes qui activent des réactions } \\
& \text { inappropriées.» }
\end{aligned}
$$

Tiraillées entre la recherche et les besognes, Marie oublie de manger,de dormir. Inconsciente de sa folie de science, elle use et abuse de forces qui s'épuisent, son poids baisse or les quatre années de hangar, Marie perd sept kilos donnant à son visage une pâleur.

N'oublions pas de signaler que l'existence de deux savants serait complètement heureuse si les chercheurs pouvaient donner toutes leurs forces dans leur pauvre hangar. Hélas, Ils doivent supporter d'autres luttes dont ils ne sortent que vaincus. Cinq cents francs couvraient les dépenses du ménage cependant après la naissance d'Irène, les gages d'une servante ainsi d'une nourrice ont fortement rongé le budget. En plus, Marie a logé le pavillon du boulevard Kellerman : quatorze cents francs.

Poussé par le besoin, Pierre demande et obtient un poste de répétiteur à l'École Polytechnique où il reçoit deux mille cinq cents francs par an. Changeant une tâche contre une autre mieux payée, il quitte l'École Polytechnique pour un poste dans

\footnotetext{
1) Curie, (E) Madame Curie, opcit., P.188

2) Jean-Baptiste , http;//www.reussitepersonnelle.com/maitrisedesoi 18/10/ 2015
}

Vol. 48 
l'enseignement du P.C.N, à l'annexe de la Sorbonne, rue Cuvier. Quant à Marie, elle reçoit une lettre de nomination du vicerecteur de l'École Normale Supérieure de Jeunes filles de Sèvres, près de Versailles où elle a posé sa candidature de professeur Ainsi le budget est équilibré pour longtemps.

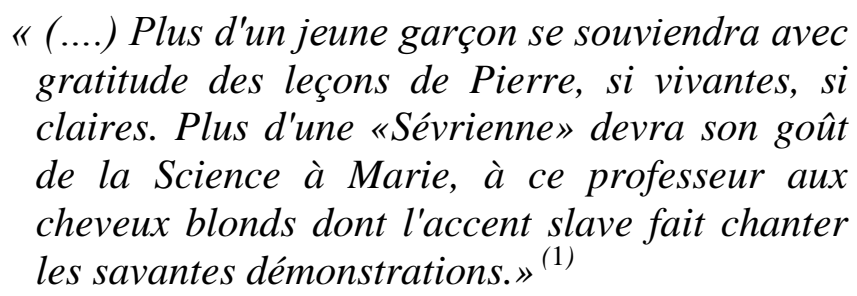

\section{Les premières découvertes}

La première sollicitude de la candide au doctorat est de jauger le« pouvoir d'ionisation» des rayons de l'uranium. La meilleure méthode employée qui incarnera la clé du succès de ses expériences a été autrefois inventée par l'étude d'autres phénomènes par deux frères physiciens qu'elle connaît très bien: Pierre et Jacques Curie. L'installation utilisée par Marie se forme d'une « chambre d'ionisation», d'un électromètre Curie et en plus d'un quartz piézoélectrique. Après quelques semaines, Premier résultat: Marie acquiert la conviction que l'intensité de ce rayonnement étonnant est proportionnelle à la quantité d'uranium contenue dans les échantillons analysés et que le rayonnement n'est influencé ni par la condition de combinaison chimique de l'uranium ni par les événements extérieurs comme l'éclairement ou la température. De plus, Marie s'enfonce dans l'intimité des rayons de l'uranium, ils lui paraissent anormales d'une essence étrangère. Virer et revirer l'énigme dans sa tête, orientée vers la vérité, Marie devine et bientôt peut prouver que l'incompréhensible rayonnement est une propriété atomique et se posant une question:

1) Curie, (E) Madame Curie, opcit., P.205

Vol. 48 
"bien que le phénomène n'ait été observé qu'avec l'uranium, rien ne prouve que l'uranium soit le seul élément chimique capable de le provoquer. Pourquoi d'autres corps ne posséderaient- ils pas le même pouvoir? c'est peut- être par hasard que les rayons ont été d' abord découverts dans l'uranium,(....) Il faut, maintenant, les chercher ailleurs.» ${ }^{(1)}$

Renoncée à l'étude de l'uranium, Marie commence l'examen de tous les corps chimiques, le résultat n'est pas attendue or les composés d'un autre corps, le thorium diffusent, eux aussi, des rayons pareils à ceux de l'uranium et d'une intensité identique. La jeune physicienne avait vu clairement: le phénomène n'est aucunement l'apanage du seul uranium et il implique de lui donner une dénomination différente. C'est pourquoi Marie Curie projette le nom de radioactivité or les deux corps «l'uranium» ainsi que « le thorium» doués de cette radiance singulière s'appelleront des radio- éléments.

La radioactivité manœuvre la physicienne $\mathrm{qu}^{\prime}$ elle ne se décourage pas d'étudier constamment par la même méthode les matières les plus variés. Féminine et extraordinaire curiosité, première faculté de savant, que Marie possède au plus haut point. Au lieu de limiter ses observations aux composés simples, sels et oxydes, elle a brusquement envie de consulter la collection de minéraux de l'École de Physique et de faire subir à divers échantillons, à l'aventure, ce genre de visite douanière qu'est l'épreuve de l'électromètre.

Pierre donne son accord et choisit avec elle les parties veinées, dures ou friables, aux formes bizzares, qu'elle s'est décidée d'examiner. La jeune femme sait d'avance ce que va lui informer

1) Curie, (E) Madame Curie, opcit., P.173 Vol. 48 
l'examen des minéraux ou plutôt elle croit le savoir .Les échantillons qui ne recèlent ni uranium ni non plus thorium, se révèleront complètement «inactifs» tandis que les autres, renfermant de l'uranium ou du thorium, seront radioactifs.

Rejetant les minéraux inactifs, Marie se consacre aux autres et mesure leur radioactivité : cette radioactivité se manifeste beaucoup plus forte que celle que l'on pouvait généralement calculer d'après la quantité d'uranium ou de thorium contenue dans les produits examinés.

«-Ce doit être une erreur d'expérience ...

(...) car, devant un phénomène

inattendu, le doute est la première

réaction du savant.» ${ }^{(1)}$

Marie recommence ses mesures dix fois, vingt fois avec les mêmes produits et il implique bien de se diriger vers la vérité .D'où provient cette radioactivité outrancière ? une seule interprétation est possible : les minéraux doivent renfermer, en quantité minime, une matière beaucoup plus fortement radioactive que l'uranium ainsi que le thorium. Mais quelle matière ? la jeune physicienne répond à la question avec la formidable audace de très grands esprits. Elle projette une brave hypothèse: les minéraux émettent sûrement une matière radioactive qui est un élément chimique nouveau, inconnu!

$$
\begin{aligned}
& \text { "(...) la motivation personnelle est une des } \\
& \text { clés menant à l'épanouissement } \\
& \text { personnel.» }(2)
\end{aligned}
$$

1) Curie, (E) Madame Curie, opcit., PP.174-175

2) Jean- Baptiste, http://www.reussitepersonnelle.com/maitrise-de-soi/ $18 / 10 / 2015$

Vol. 48 
Un élément nouveau! Hypothèse séduisante.....jusqu'à ce moment, la matière radioactive n'existe que dans l'imagination de Marie et dans celle de Pierre. Mais elle y existe.

"(....) «'instant de la découverte $n^{\prime}$ existe
pas toujours. Les travaux d'un savant sont
trop ténus pour que, dans le cours de son
pénible travail, la certitude de la réussite
crépite soudain comme un éclair et
l'éblouisse de ses feux.» ${ }^{(1)}$

En une communication à l'Académie, exposée par le professeur Lippamann et s'éditée aux Comptes Rendus de la séance du 12 avril 1898, Marie Sklodowska déclare 1' apparente présence d'un corps nouveau radioactif dans les minérais de pechblende.

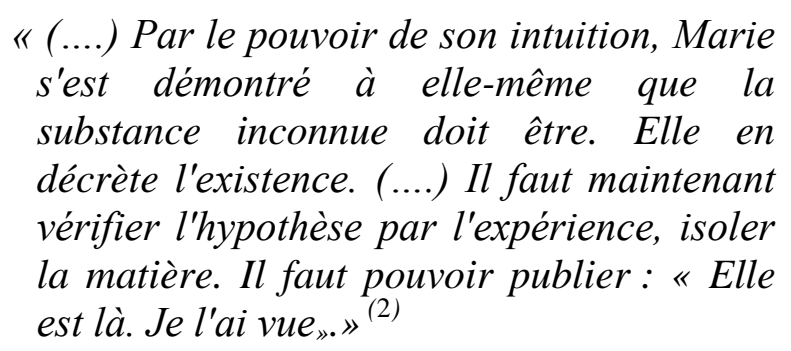

L'importance primordiale du résultat obtenu a repoussé Pierre Curie à laisser ses études sur les cristaux et à accoupler ses efforts à ceux de Marie afin de s'emparer de la matière nouvelle. C'est ainsi que dans l'immensité d'une mission impérieuse implique une collaboration, un grand physicien apparaît à côté de sa femme la jeune physicienne d'une manière qui sera impossible de discerner la part de chacun.

\footnotetext{
1) Curie, (E) Madame Curie, opcit., P. 175

2) Ibid., P.176

Vol. 48 
Les Curie fouillent la matière très active dans un minéral d'urane, la pechblende, dans son état de nature, s'est manifestée quatre fois plus radioactive que l'oxyde d'urane pur qu'elle renferme. L'élément nouveau s'y existe en quantités bien minimes pour s'enfuir à l'attention de deux savants à la sévérité des analyses chimiques.

\section{«(.....) Pierre et Marie estiment que le minerai doit recéler au maximum un pour cent de la substance nouvelle. Ils se disent que c'est bien peu ... Quelle consternation serait la leur s'ils savaient que l'élément radioactif inconnu ne figure même pas, dans la pechblende, pour un millionième !» ${ }^{(1)}$}

Avec patience, les deux physiciens commencent leur fouillement à l'aide d'une méthode de leur invention basant sur la radioactivité : ils dissocient, par les procédés ordinaires de l'analyse chimique, tous les corps dont est renfermée la pechblende, puis ils mesurent la radioactivité de chacun des produits recueillis.

La radioactivité s'accumule essentiellement dans deux fractions chimiques de la pechblende. Pour $\mathrm{M}$ et Mme Curie, c'est le signe de l'existence de deux éléments nouveaux distincts. En juillet 1898, ils sont en mesure de déclarer la découverte d'un de ces deux éléments, de suite, il faut lui trouver un nom. Après un moment de réflexion, le cœur de Marie se jette vers sa patrie opprimée; elle songe, confusément, que cet événement scientifique sera publiée chez les oppresseurs en Russie,en Allemagne, en Autriche ce qui l'amène à appeler ce nouveau élément Polonium. Ajoutons encore qu'avant la note écrite pour l'Académie sur la nouvelle matière, Marie en avait envoyé le manuscrit dans son pays natal, à Joseph Boguski, directeur du

1) Curie, (E) Madame Curie, opcit., PP.178-179

Vol. 48 
laboratoire du Musée de $1^{\prime}$ Industrie et de l'Agriculture où elle essaya jadis ses premières expériences.

La communication fut éditée à Varsovie dans une " revue mensuelle de photographie» appelant Swiatlo, presque en même temps qu'à Paris. Aussi une note remarquable a été écrite par Marie, Pierre Curie et un collaborateur appelé G.Bémont vouée à $1^{\prime}$ Académie des Sciences et éditée dans les Comptes Rendus de la scéance du 26 décembre 1898 annonçant l'existence d'un deuxième élément chimique radioactif dans la pechblende.

"(...) la nouvelle substance
radioactive renferme un élément
nouveau, auquel nous proposons de
donner le nom de RADIUM.» 1 (1)

Jusqu'à ce moment, personne n'a vu ni connu le radium or les chimiques fidèles à leurs théories déduisent: Pas de poids atomique signifie pas de radium. Pour présenter le Polonium et le Radium, pour témoigner à l'Univers l'existence de ces deux éléments, Monsieur et Madame Curie doivent travailler pendant quatre ans afin de les obtenir purs.

Pour séparer les nouveaux métaux, il va falloir traiter d'énormes quantités de matière première ce qui amène à se poser trois questions inquiétantes :

Comment se procurer une quantité suffisante de minerai? Dans quel local effectuer le traitement?

Avec quel argent payer les frais inévitables du travail? ${ }^{(2)}$

1) Curie, (E) Madame Curie, opcit., PP.182-183

2) Ibid., P.185

Vol. 48 
La pechblende, où se dissimulent le Polonium ainsi que le Radium, est un minerai précieux traité aux mines de SaintJoachimsthal, en Bohême, pour en extraire les sels d'urane employés dans 1'Industrie du verre. Les tonnes de pechblende coûteraient trop cher pour le couple Curie !..... Or si la pechblende, à l'état de nature, est coûteuse, ses résidus, après traitement n'ont qu'une valeur infime.

Par la demande à un collègue autrichien d'un soutien des directeurs de ces mines il ne serait peut- être difficile d'obtenir une bonne qualité de ces résidus. De même, il faut acheter la matière première en payant son transport à Paris. Si Marie et Pierre, qui se trouvent sur le chemin d'une découverte énorme, demandaient de l'Université de Paris ou du Gouvernement une subvention pour avoir des résidus de pechblende « on leur rirait au nez.» ${ }^{(1)}$

Quoi qu'il en soit, leur lettre se gaspillerait dans les dossiers de plusieurs bureaux et il leur faudrait des mois et des mois avant d'obtenir une réplique, probablement adverse. Des traditions et des règles de la Révolution Française, créant le système métrique, institua l'École Normale, et en maintes conditions encouragea les sciences, l'Êtat ne semble avoir mémorisé, après plus d'un siècle, que les regrettables paroles proférées par «Fouquier-Tinville à l'audience où Lavoisier fut condamné à la guillotine : LLa République n'a pas besoin de savants»» (2)

Par miracle, les résidus des récentes extractions d'uranium n'ont pas été disséminés, la matière inutile a été amoncelé dans un terrain planté de pins bordant la mine de Saint- Joachimsthal. Grâce à l'intervention du professeur suess et en plus

1) Ibid., P. 186

2) Curie, (E) Madame Curie, opcit., P.186

Vol. 48 
l'Académie des Sciences de Vienne, le gouvernement autrichien, étant le propriétaire de cette usine d'Êtat, a conclu de mettre gratuitement une tonne de résidus à la disposition des deux savants qui ambitionnent en avoir besoin. Si ceux-ci souhaitent recevoir ensuite une quantité plus grande de matière, elle leur sera attribuée par la mine aux meilleures conditions.

Un matin, une lourde voiture à chevaux, pareille à celles qui charrient le charbon, s'arrête rue Lhomond, devant l'Ecole de Physique. On a averti Pierre et Marie, les deux se précipitent au dehors, en tabliers de laboratoire. Pierre garde son calme familier mais Marie, à la vue des sacs, sans patienter, veut ouvrir un des sacs et contempler son trésor. Elle coupe les cordes, plonge ses mains dans la matière brune et terne auquelle des pins de Bohême sont restés mêlées là où se cache le radium qu'elle va extraire.

«J'ai été amenée à traiter jusqu'à vingt
kilogrammes de matière à la fois, écrit- elle
ce qui avait pour effet de remplir le hangar
de grands vases pleins de précipités et de
liquides. C'était un travail exténuant que de
transporter les récipients, de tranvaser les
liquides et de remuer, pendant des heures, la
matière en ébullition dans une bassine de
fonte.» ${ }^{(1)}$

C'est grâce à son cerveau ainsi que ses muscles que des produits de plus en plus riches en radium, ont été placé sur les vieilles tables du hangar Marie repousse la fatigue, la difficulté et même les obstacles qui entravent sa tâche. Elle se heurte quelquefois à des phénomènes ou à des méthodes qu'elle connaît mal et pour lesquels il lui faut, un arrêt, afin de se documenter. Les deux

1) Curie, (E) Madame Curie, opcit., P.189 Vol. 48 
physiciens étaient absorbés complètement par le nouveau domaine qui s'ouvrait devant eux.

En 1902, quarante- cinq mois après le jour où les Curie proclamaient l'existence probable du radium, enfin, Marie réussit à préparer un décigramme de radium pur en faisant une première détermination du poids atomique de la substance nouvelle qui est de 225 . Il ne reste qu'aux chimistes sceptiques que de s'incliner devant la surhumaine obstination de cette femme savante.

Selon Arendt «Eminents parmi les modes existentiels du direla- vérité sont la solitude du philosophe, l'isolement du savant et de l'artiste, l'impartialité de l'historien et du juge, et l'indépendance du découvreur de fait, du témoin et du reporteur» ${ }^{(1)}$

Au cours des années 1899 et 1904 ,les Curie publient tantôt dissociés, tantôt en participation avec tel de leur collègues, trente-deux communications scientifiques, les documents de ces notes sont hérissés de graphiques et de formules qui effrayent le profane. Chacun d 'eux pourtant, reflète une victoire.

" (....) En lisant la sèche énumération des communications les plus importantes, Accordons une pensée à ce qu'elles recèlent de curiosité, d'obstination, de génie.

- Sur les effets chimiques des rayons de radium. (Marie Curie et Pierre Curie, 1899 )

- Sur le poids atomique du baryum radifère. (Marie Curie, 1900)

- Les nouvelles substances radioactives et les rayons qu'elles émettent. (Marie Curie, Pierre Curie, 1900 (...)

- Sur les corps radioactifs. (Marie Curie et Pierre Curie, 1901)

- Sur le poids atomique du radium. (MariCurie,1902)

- Sur la mesure absolue du temps. (PierreCurie1902)

1) Arendt,(H) Responsabilité et jugement, Paris, Payot, 2005, P.331 Vol. 48 
- Sur la radioactivité induite et sur l'émanation du radium. (Pierre Curie, 1903)

- Recherches sur les substances radioactives. (Marie Curie, 1903) ${ }^{(1)}$

Née en France, la radioactivité séduit d'une manière rapide l'étranger. Dès 1910, des lettres, des demandes de renseignements d'Allemagne, d'Angleterre, d'Autriche et Danemark, signées des grands noms célèbres de la science, arrivent Lhomond. Dans de nombreux pays, des chercheurs se lancent à la quête d'éléments radioactifs inconnus ; ils souhaitent parvenir à de nouvelles découvertes.

Il n'y a pas longtemps que les savants considéraient que notre Univers composé de corps bien définis, d'éléments stables à jamais or voici qu'à chaque seconde qui passe, des particules de radium expulsent d'elles-mêmes des atomes de gaz hélium en les projetant au dehors avec une force immense...Le résidu de cette minuscule et effroyable explosion que Marie appelle « le catalysme de la transformation atomique » est un atome gazeux d'émancipation, se transformera lui-même en un autre corps radioactif lequel se modifiera à son tour ! De cette façon, il est à déduire que les radio- éléments constituent de bizarres, de cruelles familles où chaque organe est crée par la transformation immédiate de la substance mère le radium est un «descendant $» d e$ l'uranium, le polonium est un descendant du radium.Ces corps, à chaque instant crées, se détruisent eux-mêmes, suivant des lois éternelles : chaque radio élément perd la moitié de sa substance, en un temps qui est toujours le même et que l'on nommera sa "période». ${ }^{(2)}$

Stable en apparence, la matière abrite des naissances, des chocs, des suicides, des meurtres. Elle dissimule des épreuves

1) Curie, (E) Madame Curie, opcit., PP.212-213

2) Ibid., P.216

Vol. 48 
soumises à d'implacables destins. Elle abrite la vie et la mort tels sont les actes que la découverte de la radioactivité a révélés.Dernier et touchant miracle: le radium peut offrir quelque chose pour le bonheur des êtres humains. Il deviendra leur partenaire contre un mal terrible, le Cancer. En supprimant les cellules malades, le radium guérit des lupus, des tumeurs. Cette thérapeutique prendra le nom de Curiethérapie. En outre, des praticiens français ( Daulos, Wickam. Dominici, Degrais ) mettent en pratique avec succès les premiers traitements à des malades, en employant des tubes d'émanation du radium précis prêtés par Marie et Pierre Curie. Or depuis que les résultats thérapeutiques du radium sont connus, l'on fouille partout les minerais radioactifs. Des exploitations sont en projet dans plusieurs pays en particulier la Belgique et l'Amérique cependant les usines ne pourront produire ce «fabuleux métal » que quand les ingénieurs sacheront le secret de la préparation du radium pur.

Pierre Curie hésite entre deux choix : le premier décrire les résultats des recherches renfermant les procédés de purification ou bien de se considérer comme les inventeurs du radium c'est à dire garantir les droits sur sa fabrication dans le monde entier. Quant à Marie, elle rejette l'idée du gain en disant : « (...) (...)Si notre découverte a un avenir commercial, c'est là un hasard dont nous ne saurions profiter.Et le radium va servir à soigner les malades...Il me paraît impossible d'en tirer un avantage.(...) ce serait contraire à l'esprit scientifique.» $^{(1)}$

Si la Suisse était la première à offrir aux Curie une situation digne de leur mérite,C'est d'Angleterre que leur vinrent les premiers honneurs. En Novembre1903, une lettre est envoyée à $\mathrm{M}$ et $\mathrm{Mme}$ Curie annonçant que la Société Royale de Londres, à

1) Curie, (E) Madame Curie, opcit., P.223

Vol. 48 
son tour, leur marque son estime par une de ses plus hautes récompenses : la médaille Davy.

A la « réunion solennelle générale» du 10 décembre 1903, l'Académie des Sciences de Stockholm a annoncé publiquement que le prix de Nobel de physique est descerné par moitié à Henri Becquerel, par moitié à M.et Mme Curie, pour leurs découvertes sur la radioactivité. Surchargés de travail, Pierre et Marie ont reflué devant ce long trajet alors le ministre de France a reçu en leur nom, des mains du roi, diplộmes et médailles d'or.

\section{«DémythifierMarie-Curie, la scientifique française aux deux prix Nobel( de physique en1903, avec son mari, Pierre, et de chimie en1911, tel est le propos de sa petite- fille» ${ }^{(1)}$}

Il est à noter qu'à titre de prêt, Marie envoie vingt mille couronnes autrichiennes aux Dluski pour aplanir la mise en marche de leur sanatorium. Et le reste de cette petite fortune, qui augmentera des cinquante mille francs du prix Osiris attribué par moitié à Marie Curie et à Edouard Branly, est légitimement divisé en rentes françaises et en obligations de la ville de Varsovie. En plus, quelques autres dépenses excessives retrouvées dans son cahier de comptes noir : des prêts au frère de Pierre, à ses sœurs-générosité que l'extrême discernement de leurs bénéficiaires diminuera souvent à des proportions modestes. Des contributions à des sociétés scientifiques, des dons à des étudiants polonais, à une amie de jeunesse, à des garçons de laboratoire, à une sévrienne en état de pauvreté. Aussi à son professeur de Français établie, mariée en Pologne

1) Payot Marianne, www.lexpress.fr/culture/livre/marie-curie-portrait-d-unemere, P.2 -966006 
et dont son grand rêve est de revoir son pays natal Dieppe alors, Marie lui écrit en l'invitant en France, la reçoit dans sa maison et paye son voyage de Varsovie à Paris et de Paris à Dieppe.Durant toute sa vie, Marie a décidé d'aider ceux qui ont besoin d'elle. Elle désire le faire selon ses moyens, afin d'être capable de continuer toujours.

La célébrité devrait apporter aux Curie quelques avantages : la chaire, le laboratoire, les collaborateurs et les crédits tant souhaités. Mais rien de ces bienfaits. Au surplus, Nous tâtons à l'une des raisons essentielles de l'agitation de deux savants. La France est le dernier pays où leur valeur a été reconnue et il n'a pas fallu moins que la médaille Davy et le prix Nobel pour que l'université de Paris attribuât enfin une chaire de physique à Pierre. Les deux physiciens en éprouvent de l'affliction, les récompenses provenues de $1^{\prime}$ Etranger accentuent les conditions attristantes dans lesquelles ils ont fait réussir leur découverte, conditions qui paraissent loin de changer Rendant hommage à la seule institution qui ait excité et soutenu leurs efforts: L'Ecole de physique.

Quant à la renommée, la répugnance qu'elle inspire aux Curie a d'autres racines que leur passion du travail ou que leurs affres du temps perdu. Pierre déteste les hiérarchies, lesclassements. Les décorations que souhaitent les grandes personnes semblent superflues que les médailles accordées aux enfants des écoles. Son indifférence a certes une influence profonde sur Marie.Mais ce n'est pas ni pour l'imiter ni pour l'obéir que, durant toute sa vie, elle se détournera aux témoignages d'admiration. Une irrésistible réserve, une crispation accablante la figent dès qu'elle doit braver la foule en provoquant même des troubles qui vont jusqu'au vertige, jusqu'au souffrance physique. dépassait nos forces et qui nous avait été 


\begin{abstract}
imposé par les conditions matérielles peu satisfaisantes de notre travail, fut augmentée par l'invasion de la publicité, écrira Marie plus tard. ${ }^{(1)}$
\end{abstract}

Portant sur son dos le poids du travail, de son foyer, de sa maternité, de son enseignement, Mme Curie s'évolue telle qu'une acrobate sur son chemin difficile.Elle n'a pas une seconde à perdre pour jouer les femmes célèbres.Ayant accompli ensemble une grande œuvre, Pierre et Marie accueillent la gloire d'une façon différente.

Les deux époux traversent cette épreuve en restant unis dans leur distance des honneurs. rappelons donc ces quelques mots sensibles d'Eve Curie.
"(...) Que cette magnifique aventure ait fait constamment souffrir son hérö̈ne.(....) j'eusse donné beaucoup pour découvrir au bas d'une lettre ,à travers une confidence (...) un cri ou un soupir de victoire.(...) Marie, promue au rang de "la célèbre Madame Curie» sera encore heureuse parfois, mais seulement dans le silence de son laboratoire ou dans l'intimité de sa maison. Jour après jour, elle se fera plus terne, plus effacée, plus anonyme, pour échapper à ceux qui veulent la traîner sur la scène, pour n'être pas cette vedette dans laquelle elle ne saurait se reconnaitre.» ${ }^{(2)}$

Comme Marie, Irène Joliot-Curie, mettait au monde une génération de scientifique. Sa fille, Hélène, a épouser le petit-fils de Paul Langevin, Michel, lui- même physicien nucléaire. Hélène Langevin- Joliot ingénieur, comme son père, de L'Ếcole Supérieure de physique et de chimie industrielles de la ville de Paris, participa au lancement1957 de L'Institut de Physique

\footnotetext{
${ }^{1)}$ Curie, (E) Madame Curie, opcit., PP.238-239

${ }^{2)}$ Curie, (E) Madame Curie, opcit., P.241

Vol. 48 
Nucléaire d'Orsay créant à l'initiative de sa mère juste avant le décès de celle- ci.

Directrice de recherches émérite au C.N.R.S, Hélène Langevin s'est consacrée à la préservation du patrimoine scientifique d'une côté et la place des femmes dans la Science de l'autre côté, l'application de la physique nucléaire au domaine médical beaucoup plus pour le diagnostique que pour le traitement des maladies, que pour les méthodes les plus évoluées de l'imagerie médicale a compensé, pour cette femme de paix, les effets négatifs de quelques- uns des aspects du nucléaire. Elle a été présidente de la commission de physique nucléaire du comité national du C N R S de 1982 à 1986 en plus, elle a été membre du conseil scientifique de l'office parlementaire pour les choix scientifiques et techniques de 1985 à 1992.

Le frère d'Irène Joliot, Pierre, est devenu directeur de la recherche au CN R S EN 1974 prenant, entre autres, la direction du Département de Biologie de L'Ếcole Normale Supérieure de 1987 à 1992, avant d'être président au conseil scientifique de L'École Normale Supérieure de Lyon. Professeur honoraire au collège de France, ancien titulaire de la charie de bioénergétique cellulaire, spécialiste de la photosynthèse encore membre de L'Académie des Sciences et de la National Academy américaine, il a obtenu le prix du commissariat à L'Ênergie atomique en 1980 en plus, La médaille d'or du C N R SEN 1982.

Il s'est obsédé souvent de l'avenir de la recherche en faisant, en écrivant plusieurs conférences sur ce sujet. Quant à Yves Langevin, le fils d'Hélène et de Michel, celui-ci était astrophysicien à Orsay. Il a spécialement étudié la minéralogie de la planète Mars ainsi que les causes possibles pour lesquelles son évolution et celle de la Terre ont divergé avec rapidité sans éliminer l'hypothèse d'une exobiologie martienne initiale. 
Cette œuvre, cette rigueur et cet engagement hérités surtout de Marie Sklodowska, qui fut une des premières femmes à vivre la Science comme un métier en mettant un but à aboutir avec honnêteté et en restant toujours un exemple vif pour tous les chercheurs de la Science.

\section{Conclusion}

Grâce au roman d'Eve Curie, nous avons lu une riche biographie passionnée animé par un amour familial. Cette biographie complète d'autres biographies écrites visant à s'adresser aux esprits seulement quant à celle de notre écrivaine s'attache à interpeller âme et esprit.

Aussi cette biographie inscrit son contexte dans des genres qui s'entremêlent. Le tragique et le pathétique, le didactique et le polémique, le réalisme et le poétique sont autant de facettes d'une figure remodelable à l'infini. Ses multiples éléments tressés procurent à l'écriture biographique toute la richesse d'une personnalité aussi étrange que son sujet : la vie.

Eve curie doit à sa famille polonaise et particulièrement à la sœur aînée de sa mère, Madame Dluska, qui était sa plus douce compagne, des lettres et des témoignages spontanés. Des papiers personnels, de notes biographiques concises laissés par la savante Marie Curie, de multiples textes officiels, les récits ainsi que la correspondance d'amis français et polonais qu'elle ne saurait suffisamment remercier, en plus, les souvenirs de sa sœur, Irène joliot-Curie, de son beau-frère Frédéric Joliot et ses miens, qui l'ont aidé à évoquer les années les plus récentes.

Au début, Eve Curie a essayé de nous dessiner deux portraits de Mania Sklodowska, l'un à l'étape de l'enfance, ayant le cour aimable, délicat, gai dans ses jeux avec sœurs, frères, dans ses études où les professeurs affirment qu'elle est «remarquablement douée », le deuxième, étant le plus grave, à l'étape de l'adolescence, où des êtres aimés se sont disparus qu'une mémoire sensible rappellera jusqu'au dernier souffle. 
Malgré tout ce qui s'est passé autour d'elle, Mania n'avait qu'une seule idée obsédante : Apprendre.

Sa patience s'est montrée évidemment en respectant la décision de son père de vivre à la campagne chez des parents de province en donnant des leçons et en supportant la lassitude ainsi que l'humiliation qui attendait une répétitrice afin de résoudre le problème financier de son père.

Son courage s'est manifesté également par son adhésion aux cours de «l'université volante » donnés aux jeunes sans avoir peur de la prison en vue de développer leur culture Aussi, des leçons offertes aux employées d'un atelier de- prêt -À- porter, en constituant série par série, une petite bibliothèque, à la volonté des ouvrières. De plus, des cours clandestins de polonais donnés à de petits élèves fils et filles de servantes, de paysans, d'ouvriers visant d'exciter ces jeunes cerveaux à la beauté de la langue et de l'histoire nationale. Toujours présente en étant «l'idéaliste positive», stimuler de combattre, de se rendre profitable pour les humbles.

Non seulement se préoccuper de son pays asservie mais Marie s'est sentie responsable de l'avenir de ses aînés. Ayant tant réfléchi jusqu'à trouver un moyen pour réaliser le grand rêve de sa sœur en se plaçant comme institutrice avec quatre cents roubles par an pour lui permettre de débuter ses études à Paris.

La patience et le courage sont deux facteurs essentiels conduisant à l'art de se maîtriser, de s'adapter aux difficiles circonstances, de les surpasser décidant de passer deux licences celle de physique et celle de mathématiques. Une étude sur les propriétés magnétiques de divers aciers commandée par la Société pour l'Encouragement de l'Industrie National l'a conduit à faire la connaissance de Pierre à l'École de Physique et de Chimie, rue Lhomond. Celui - ci était attiré par cette jeune femme étrangère, charmante réussissant après plusieurs demandes de mariage d'être sa femme. 
Durant les jours de noce se tisse une des plus belles liaisons qui aient jamais lié un homme et une femme. "Deux cours se battent à l'unisson (....) deux cerveaux de génie s'accoutument à penser ensemble. »" Et comme maître de soi signifie un savoir- faire qui s'instruit, s'expérimente durant toute la vie, le choix entre la vie familiale et la carrière scientifique ne se passait même plus à la tête de cette femme savante, elle a réglé de «mener de front »l'amour, les maternités, la science, à ne jamais tromper passion et résolution et elle y réussit.

Sa découverte d'un corps magique, le radium n'est pas uniquement la naissance d'une nouvelle science, d'une nouvelle philosophie: elle offre aux hommes le moyen de soigner une maladie horrible. «(...) (...) Aux blessés de la guerre, elle donne son dévouement et sa santé. Plus tard, elle donnera ses conseils, son savoir, et chacune des heures de son temps à ses élèves, à de futurs savants venus de toute les parties du monde. $»^{2}$

Ayant raconté les petits et les grands détails de la vie de la fée de la science, d'une femme rare, d'un génie exceptionnel, nous avons vécu avec elle cette vie dure et difficile qui ne l'a jamais déçue. De lire et relire cette laborieuse biographie, nous nous trouvons toucher par des milliers et des milliers de moments de peine ainsi que de joie. Nous avons tant et plus pleuré de même nous avons chanté la gloire.

À mon avis, Eve curie a atteint son but en voulant que les lecteurs de cette biographie ne s'arrête pas de distinguer, au milieu des événements précaires d'une existence, ce qui, chez Marie curie, est plus exceptionnel que son œuvre ou que l'original de sa vie : la stabilité du caractère, l'effort obstiné, implacable du savoir; le sacrifice d'un être qui est capable de tout donner sans attendre de prendre ni même recevoir; l'âme enfin, dont rien n'arrive à corrompre sa pureté.

${ }^{1)}$ Curie, (E) Madame Curie, opcit., p.158.
2) Ibid., p.7.

Vol. 48 
Il nous reste de prier aux traducteurs du monde entier de s'intéresser à la vie de ces génies, de les traduire en différentes langues pour profiter de plus en plus du long trajet de ces êtres supérieurs débutant sans aucun doute du tourment, des difficultés arrivant à la victoire et à la gloire. 


\section{Bibliographie}

\section{Corpus :}

Curie (E) , Madame Curie, Hachette S.A Buenos Aires.

\section{II. œuvres Consacrées à Marie Curie :}

- Curie (E ), Madame Curie, Paris, Gallimard, 1938.

- Curie (M) , la Radiologie et la Guerre , Paris, Librairie félix Alcan , 1921.

- Grioud (F) , une femme honorable, libraire Arthème fayard, 1981.

- Joliot- curie (I) , Marie Curie , ma mère in Europe , 108, Paris, les Editeurs français réunis , 1954.

- Lemire (L), Marie Curie , Perrin , 2001.

- Radvanyi (P) , les Curie, Pionniers de L'atome, Belin , 2005.

- Regaud (C) Marie sklodowska - Curie , Paris , Fondation Curie , 1934.

- Reid (R), Marie Curie derrière la légende , Seuil , Paris , 1979.

\section{Ouvrages Généraux :}

- Arendt (H) , Responsabilité et jugement, Paris , payot , 2005 .

- Jaurès (J), Discours a la jeunesse ALbi-1903.

- Jankélévitch (V), Traité des vertus , II ,v.1, ch2 "le courage et la fidélité" Paris , champs Flammarion , 1985

- Maillard (M), L' autobiographie et la biographie , Nathan / VUEF - 2001. 


\section{Liste des liens de sites internet :}

-http://www.pasteur web . org /Etudes/ le\%.2520 fruit 2520 de 25201\%_27Esprit / la .patience .htm.

-http://www. eveil de la conscience . ca / orloff 175208$18 / 10 / 2015$.

-http://www. messager spirituel . com / la cledela maitrise de soi. htm 18/10/2015.

-http://www. philopol . ulg . ac. bc / telecharger / .. / courage.

-https://www. reussite personnelle .com / maitrise-de-soi/ $18 / 10 / 2015$.

-http://www. messager spirituel.com/L'art de Diriger notre Esprit 9/7/2016.

-www.lexpress. Fr/ culture / livre / marie-curie portrait - d une - mere.

-http://wwwmusee.Curie.Fr/public/livre-famille-aux-5-prixnobel 29/4/2015.

-http://www.marie samuse . over - blog.com / article - madame - curieeve - curie - 84705109 .htm 29/4/2015.

-http://www. liberation. Fr / livres / 2003/06/19une experience - inedite - de- marie - curie - 437. 29/4/2015.

-http://www. societe chimique de france. Fr/ produit - du - jour / curie - joliot - Langevin - une fami.... 29/04/2015.

-http://www.pasteur web.org / Etudes / le \% 2520 fruit 2520 de $\% 25201 \% 27$ Esprit / la maitrise de soi .htm.

-http://www. avis - de - deces . com / celebrites - decedees / 1322 / Eve- curie 29/04/2015. 